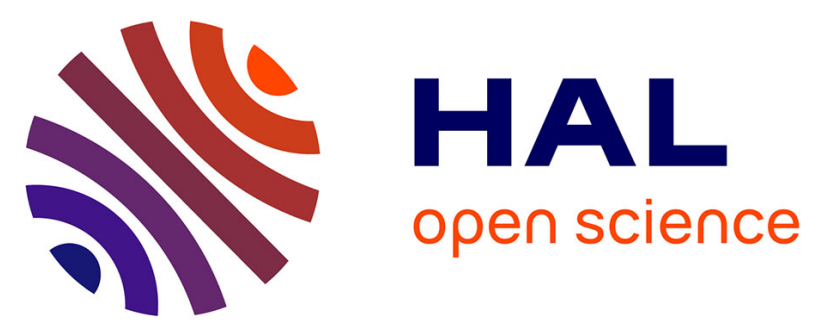

\title{
Un épisode inédit dans la genèse de l'épigraphie latine à la Renaissance: la collection d'inscriptions romaines de Nicolaus Florentius de Haarlem dans les manuscrits de Laevinus Torrentius à la Bibliothèque Royale de Bruxelles (ms 4347-50)
}

Ginette Vagenheim

\section{To cite this version:}

Ginette Vagenheim. Un épisode inédit dans la genèse de l'épigraphie latine à la Renaissance: la collection d'inscriptions romaines de Nicolaus Florentius de Haarlem dans les manuscrits de Laevinus Torrentius à la Bibliothèque Royale de Bruxelles (ms 4347-50). In Monte Artium. Journal of the Royal Library of Belgium, 2008, 1, pp.153-183. 10.1484/J.IMA.1.100292 . hal-01841000

HAL Id: hal-01841000

https://hal-normandie-univ.archives-ouvertes.fr/hal-01841000

Submitted on 3 Aug 2018

HAL is a multi-disciplinary open access archive for the deposit and dissemination of scientific research documents, whether they are published or not. The documents may come from teaching and research institutions in France or abroad, or from public or private research centers.
L'archive ouverte pluridisciplinaire HAL, est destinée au dépôt et à la diffusion de documents scientifiques de niveau recherche, publiés ou non, émanant des établissements d'enseignement et de recherche français ou étrangers, des laboratoires publics ou privés. 


\title{
UN ÉPISODE INÉDIT DANS LA GENĖSE DE L'ÉPIGRAPHIE LATINE À LA RENAISSANCE: LA COLLECTION D'INSCRIPTIONS ROMAINES DE NICOLAUS FLORENTIUS DE HAARLEM DANS LES MANUSCRITS DE LAEVINUS TORRENTIUS À LA BIBLIOTHĖQUE ROYALE DE BRUXELLES (MS 4347-50)1
}

\author{
Ginette VAGENHEIM
}

A la mémoire de Tony Hackens

Les humanistes belges ont joué un rôle primordial dans l'histoire de l'épigraphie latine comme en témoigne l'immense fortune du corpus d'inscriptions de Martinus Smetius de Westwinckel (Maarten de Smet, 1525-c. 1578) édité par Juste Lipse (1547-1606) à Leyde en 1588 (Inscriptionum antiquarum quae passim per Europam liber. Accessit auctarium a Iusto Lipsio) et celui de Janus Gruterus d'Anvers (Jean van Gruytere, 1560-1627), qui vit le jour à Heidelberg en 1603 (Inscriptiones antiquae totius orbis Romani in absolutissimum corpus redactae). ${ }^{2}$ Ce fait avait déjà été souligné par Joseph Roulez, le biographe de Smetius, qui écrivait que: "son recueil, noyau du corpus inscriptionum de Gruterus, forme le véritable fondement de la méthode épigraphique actuelle. $»^{3}$

Les manuscrits préparatoires à ces éditions, comme celui de Smetius conservé à la Bibliothèque universitaire de Leyde (MS BPL 1) que Lipse utilisa pour publier son corpus, ou les simples recueils d'inscriptions destinés à d'autres travaux de ces érudits, ${ }^{4}$ constituent également de précieux témoignages, encore trop peu étudiés, de la contribution des huma-

(1) Je remercie amicalement Lucien Reynhout qui a bien voulu m'associer au premier numéro de cette revue; je remercie Michiel Verweij qui a notablement amélioré mes transcriptions latines effectuées à partir des manuscrits de la Bibliothèque royale ainsi que toutes les personnes travaillant à la Section des manuscrits pour leur cordiale disponibilité. Je remercie aussi de tout cœur ma tante Marie-José Ngendahayo et ma fille Lucie qui m'ont permis de conduire ma recherche dans les meilleures conditions.

(2) L'histoire du corpus de Smetius est racontée par Jeanine De Landtsheer, «Inscriptions and Coins in Lipsius's works 1588-1609, » Lias 31 (2004): 119-39 et Ginette Vagenheim, «Juste Lipse et l'édition du recueil d'inscriptions latines de Martinus Smetius," dans Iam illustravit omnia. Justus Lipsitus als lievelingsatuteur van het Plantijnse Huis, ed. Jeanine De Landtsheer en Pierre Delsaerdt (Antwerpen: Vereniging van Antwerpse Bibliofielen, 2006): 45-66 = De Gulden Passer 84 (2006).

(3) Joseph Roulez, «Maarten De Smet, in Biographie nationale, Académie royale des Sciences, des Lettres et des Beaux Arts de Belgique (Bruxelles: Bruylant-Christophe, 1876), 5:767. Alphonse Roersch répétera la même chose dans son étude sur L'bumanisme belge à l'époque de la Renaissance. Etudes et portraits (Bruxelles, 1910), 139.

(4) On verra plus loin l'usage que fait Fulvio Orsini de l'inscription CIL 6.1316. 
nistes des Pays-Bas à l'élaboration de la science épigraphique. C'est ce que nous révèlent les manuscrits de Torrentius (Lievin vander Beke, 1525-95) conservés aujourd'hui à la Bibliothèque royale de Bruxelles sous les cotes MS 4347-49 et MS 4350.5 Dans le cadre de cette étude, je me limiterai à l'examen d'un groupe d'inscriptions transmis dans ces recueils qu'un érudit originaire de Haarlem, Nicolaus Florentius, vit à Rome au moment de leur découverte, entre 1561 et 1564 , et qu'il envoya à Torrentius qui à son tour fit parvenir à Stephanus Winandus Pighius (Etienne Wynants, 1520-1604) des copies de ces mêmes inscriptions que Florentius avait rédigées à son intention, comme nous le découvrons ici pour la première fois. C'est ainsi que les seules fiches autographes de Florentius connues à ce jour sont conservées dans les manuscrits de Torrentius cités plus haut et dans un recueil d'inscriptions de Pighius conservé à Berlin (Staatsbibliotheek, MS lat. fol. 61A). ${ }^{6}$ Smetius aussi bénéficiera des découvertes de Florentius, sans doute par le truchement de Pighius et plus tard Lipse aussi recevra de l'érudit qui se trouvait toujours à Rome, des inscriptions de toute l'Italie; finalement, les inscriptions de Florentius seront publiées dans le corpus de Gruterus qui se fondera tantôt sur le texte de Smetius tantôt sur celui de Lipse, si bien que la plupart de ces inscriptions connaîtront, à la Renaissance, une transmission exclusivement belge.

Theodor Mommsen, le seul à avoir examiné les recueils de Torrentius, dans le cadre de l'édition du Corpus Inscriptionum Latinarum (CIL), avait formulé l'hypothèse que l'auteur de cette collection d'inscriptions était un certain Nicolaus Florentius Hadrlemensis qui avait envoyé d'Italie des inscriptions à Torrentius (Fig. 1): "Eique videntur tribuendae esse inscriptiones ex Italia missae ad Laevinum Torrentium, quae sunt in codicibus bibl. Bruxell. 43474349. 4350. $»^{7}$ Voyons ce que nous révèle un nouvel examen de ces recueils et plus précisément des seize inscriptions qui furent envoyées de Rome à Liège, au moment où Torrentius y exerçait la charge de prince-évêque (1557-87).

\section{LE MS 4350.}

\section{CIL 6.921}

Les six fragments de l'inscription CIL 6.921 apparaissent au f. $1 \mathrm{r}$ du MS 4350 sans aucune indication (Fig. 2a). On les retrouve chez Pighius (f. 103r), qui les a recopiés dans son recueil (Fig. 2b) ${ }^{8}$ en les y faisant précéder d'une note dans laquelle il nous livre l'identité de leur auteur en même temps que la date de l'envoi: "Misit Roma Nic.(olao) Florentius kal. Aug. 1562;" il précise en outre l'endroit où les fragments furent découverts: "reperta

(5) La bibliographie sur Torrentius est rassemblée dans Marie Delcourt et Jean Hoyoux, Laevinus Torrentius, correspondance, 3 vols (Paris: Les Belles Lettres, 1950-4).

(6) Les inscriptions apparaissent aux ff. 116, 125v, 126r-v, 127, 209.

(7) CIL 6.1:54 n. 48 .

(8) La graphie particulière de la lettre «G» («Aug.(usti)») qui forme une grosse boucle dans sa partie inférieure, est une caractéristique de l'écriture de Pighius. 


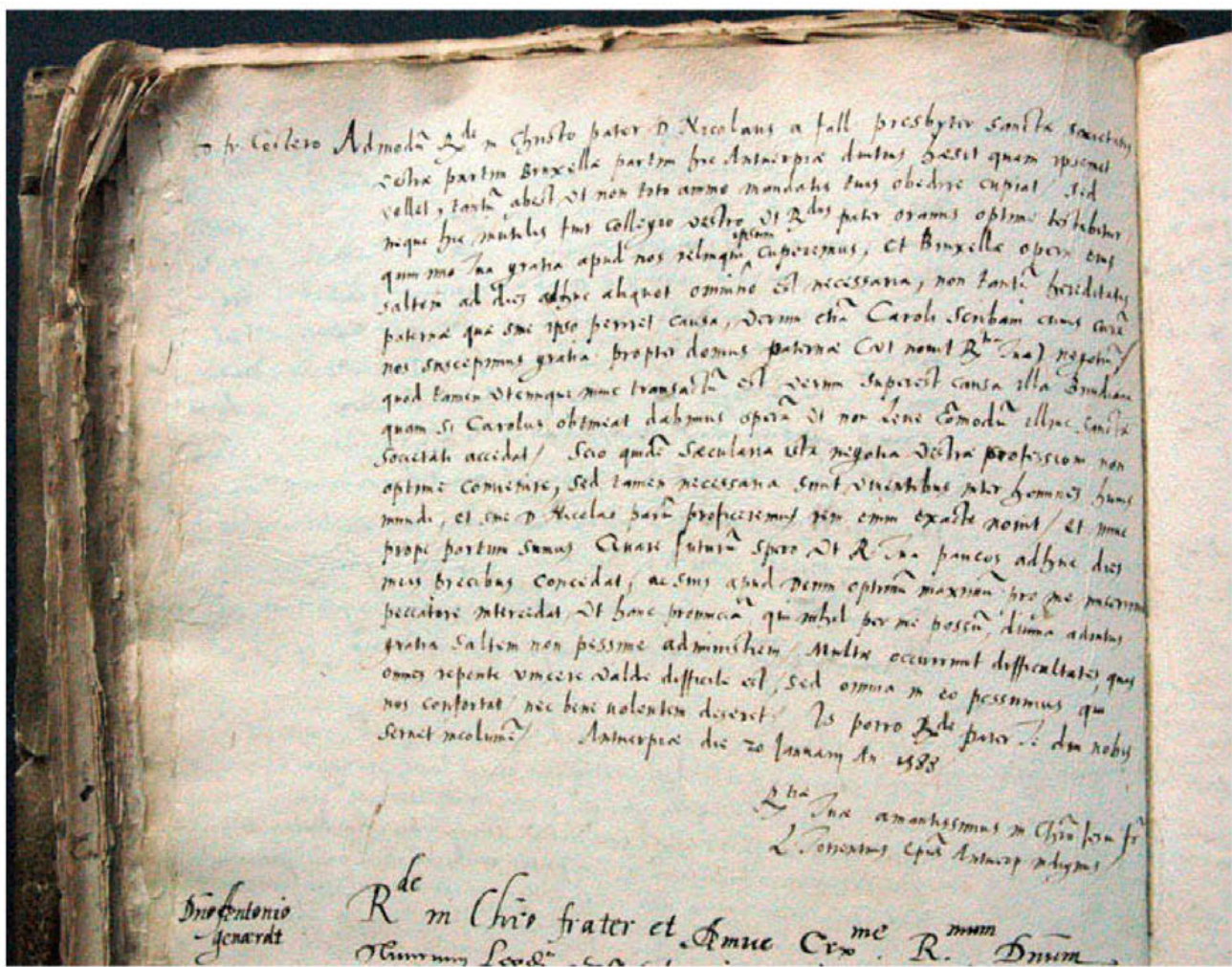

Fig. 1: Lettre datée et signée de la main de Torrentius.

in via Lata. " Smetius, dans son recueil édité par Lipse dix ans après sa mort (59, 4-6) cite lui aussi Florentius comme l'auteur de l'envoi des six fragments: "Haec a Nicolao Florentio accepimus." Comme Pighius, il nous fournit la date de leur découverte, mais indique encore plus précisément le lieu de leur trouvaille: "Repertum in via Lata ante aedes Alexandri Columnae in area vulgo 'La piazza di Zerra' appellatae; transmissumque a Nicolao Florentio anno 1562." Gruterus, qui déclare dans sa préface avoir utilisé parmi ses sources le corpus de Smetius, publiera à son tour les fragments de l'inscription (236.9 et 238.6), en citant tout naturellement le recueil du Flamand. ${ }^{9}$ Paulus Knibbius, un autre érudit belge, lui aussi encore trop méconnu, transmettra aussi l'inscription dans son corpus de Berlin (f. 17) qui constitue une autre source de Gruterus (Fig. 2c); ce dernier, qui avait eu entre les mains le recueil de Knibbius y ajouta le titre suivant: "Inscriptiones romanae quae Paulus Knibbius (regis Scotiae consiliarius intimus) partim ipse vidit, partim ex iis quae vidit Ioh. Jacobus Boissardus descripsit. ${ }^{10}$ Grâce aux renseignements combinés fournis par les témoignages de

(9) Le seul autre témoignage ancien de la copie de six fragments de l'inscription est dû à une main inconnue "manu ignota» dans l'un des recueils d'inscriptions d'Aldo Manuzio junior (1547-97) conservés à la Bibliothèque apostolique (MS Vat. Lat. 5237, f. 147).

(10) Sur Knibbius et son corpus, on consultera la brève notice dans le CIL 6.1:54 n. 48. Sur Boissard (15281602), on consultera Christian Calmer, "Un manuscrit de Jean-Jacques Boissard à la Bibliothèque Royale de 


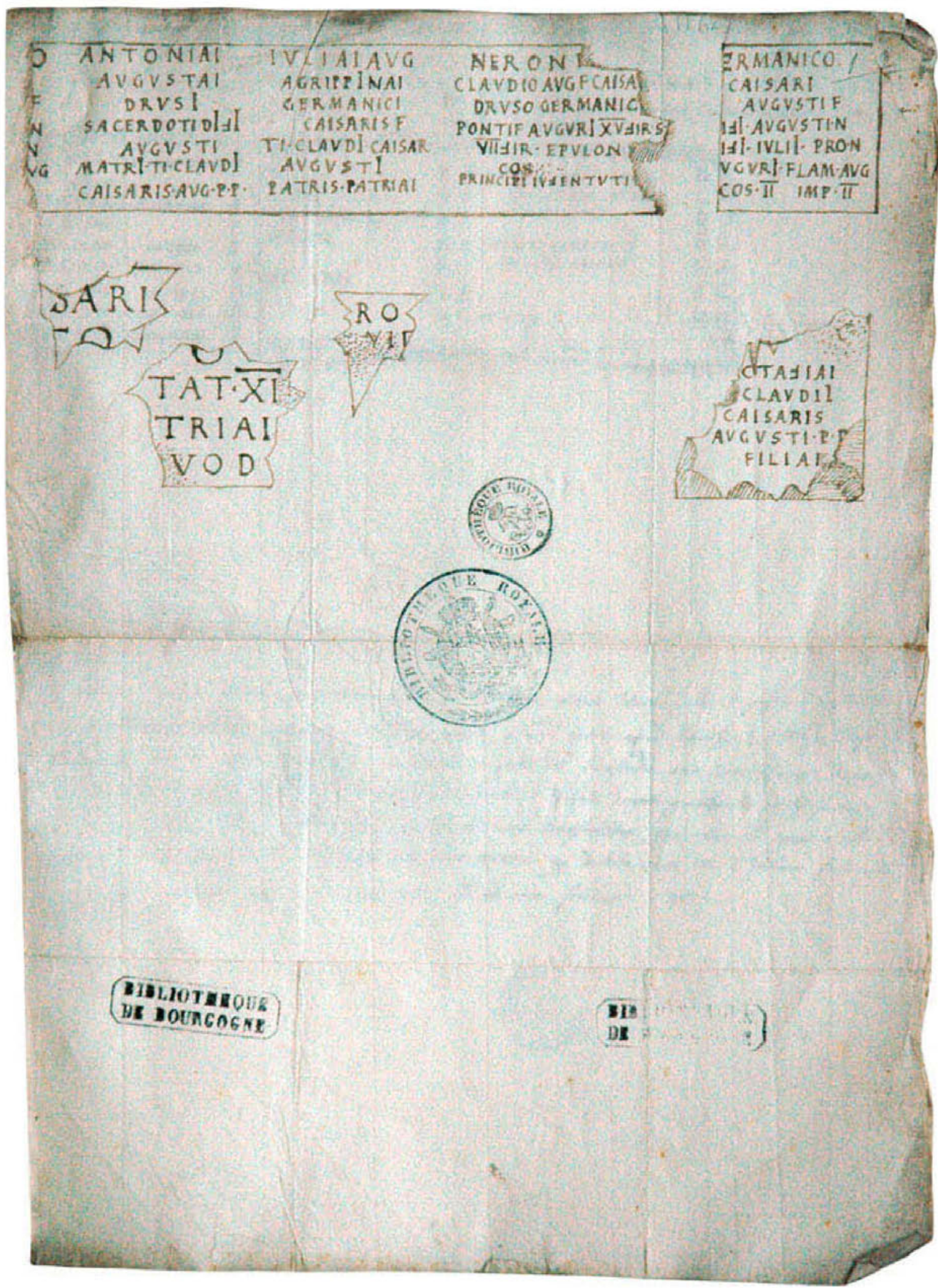

Fig. 2a: CIL VI 921 copiée par Florentius. 


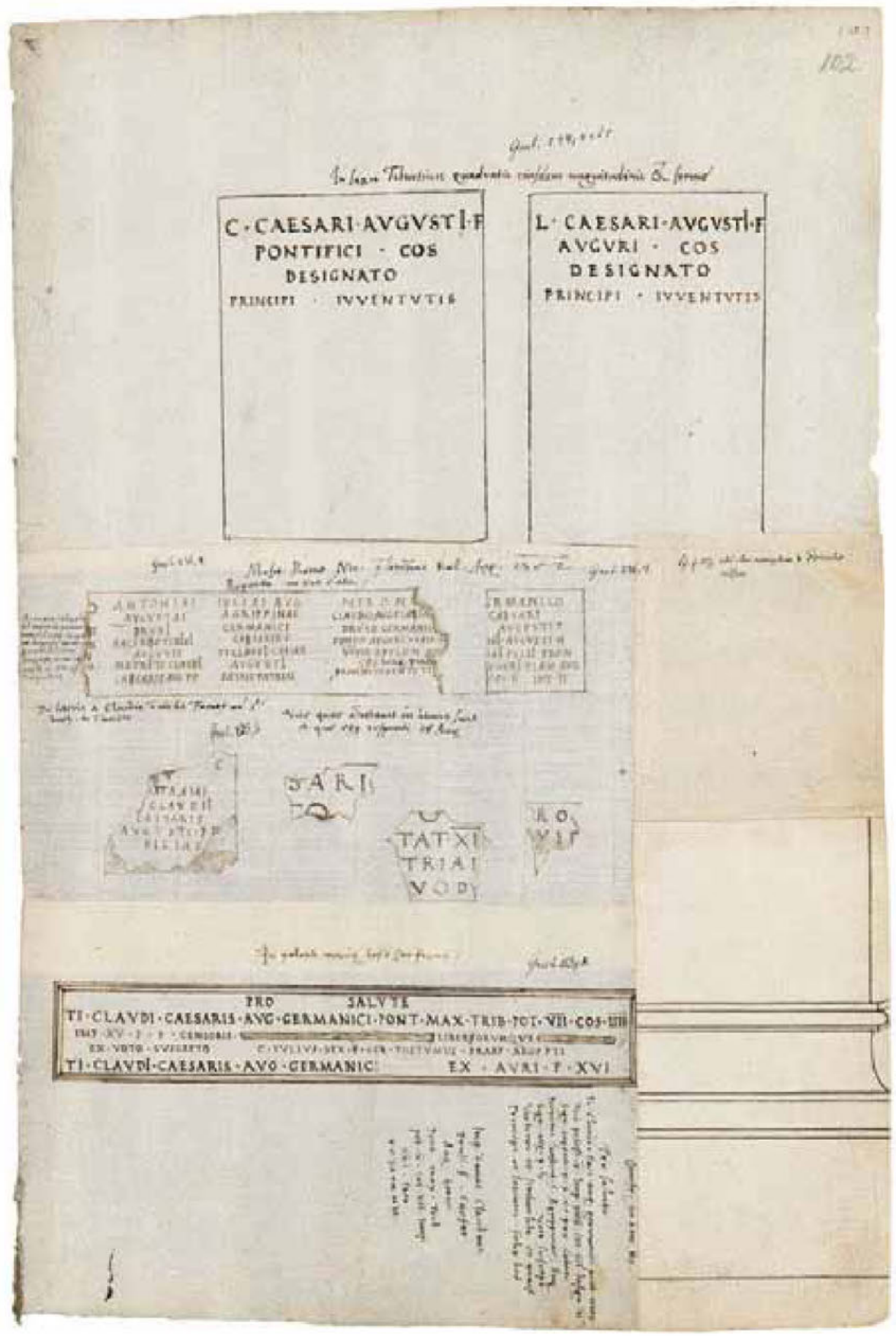

Fig. 2b: CIL VI 921 copiée par Pighius. 
In casa del Cardinal de la Valle.

IMP. CAESARI

DIVI NERVAEF

NERLAE TRALANO

AVG-GERMANCCO

DACICO PONTIFIC,

MAXIMO TREBNTC

POT.VIIt. MRIIIt, $\cos , \bar{v}$.P.

TRENS XXXV.

KVAD LIBERALTTATE

OPTIMI PRINCIPIS

COMMODA EARVM ETIAM

LOCORNM ADIECTISNE.

AMPLIATA SINTT.

IMP. CAESARI

DIVI HADRIANI

AVG.FILIO-DIVI

TRAIANI PARTHLCI

NEP. DIVI NERVAE

PRONEPOTI TITO

AELIO HADRIANO

ANTONINOANOPHO

TRIB.POT. COS. DES. II

$$
\text { SCRIBAE }
$$

ARMAMENTIET

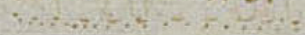

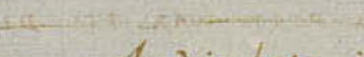

In via lata in casa del s Geouer Capizucica.

ANTONIAL IVLIAIAVE

AVOVSTAI AGRIPPINAI

DRVSI GERMANICI

SACERDOTIDIAI SCLISARIS F

AVGVSTI TICLNDI GISAR

MATRITI CLAVDI AVGVSTI

CAISARISAVERP PATKIS PATRIAE

$N E R O N$

CLAVDIA AVG:CATSA..

DRVSO GERMANIC....

PONTIF AUEYRI XVAIR.

VII VIR EPVLON

Co

PRINCIPI. TVAENTITI

M.AVRELLO CAESARI

ERMANLCO

IWP. CAESARLS.T AELI CAISARI

HADRIANI ANTONINI AVG. PII. PONT.AUA. TRIB. POT. XIIII. IMP. II. COS. III. PRF/L. DIVI HADRIANI NEP DIVI TMIIANI PARTHLCL PRONEP DIVI NERVAE AENEPOTI

TRIB, PAT. $\bar{V} \cdot \cos \cdot \bar{U}$.

$\because \because: 20 \mathrm{NENSES}$

EX.AFRICA.

- Avgustif

‥ It. ArGrstiN.

HIIIVLII.PRON.

-vgVRT. FLAAT.AUG

- OS. $\bar{l} \cdot \operatorname{lmp} \overline{\|}$.

DIVAS

FAVSTIMAE

Aro

SEXTANI

ARELATENSES.

Fig. 2c: CIL VI 921 copiée par Knibbius. 
Pighius et Smetius, on est en mesure de reconstituer le commentaire que Florentius avait envoyé à Torrentius en même temps que la copie de CIL 6.921 et qui s'est sans doute perdu au cours de la transmission.

\section{CIL 6.2294}

La copie de CIL 6.2294 (f. 2r) est précédée d'une note indiquant les mesures de la tablette de marbre dont le dessin révèle des traces de fracture; on y apprend aussi son lieu de découverte et sa date, de manière imprécise ( «il y a trois ou quatre ans»): «Fragmentum tabulae marmoreae longum uncias -20-latum uncias -6-, effossum Romae sub colle Hortulorum al. (ia)s 'de la trinită' dum ibi ante tres aut quatuor annos fundamenta domus suae cavaret monsignor del Giglio." Une seconde note, tout à fait inédite, apparaît sous l'inscription. Elle précise que le texte est un fragment des Fastes civils représentant les mois de juillet, août et septembre, comme l'a révélé la collation avec un autre fragment de Fastes conservé dans la collection d'Achille Maffei, chanoine de Saint-Pierre et qu'il sera bientôt publié par Lanfredius à Venise ou Rome. L'auteur du billet veut que le destinataire de la note envoie à Pighius une copie de l'inscription: "Fragme(n)tum superius co(n)tinet particulam fastorum civilium, nempe mensis Iulij, Augusti et Septembris ut ex collatione tabule marmoree collotiane patet q(uae) $n u(n) c$ extat apud clariss(imum) D(ominum) Achillem Maffeiu(m) q(uae) si recte memini alias $i(m)$ pressa est, et denuo ut puto ab Lanfredio aut Venetiis aut Rome brevi excudet(ur) q(uod)si Pigghius noster doctissimus $i(n)$ Belgis degat gratissimu(m) mihi fuerit si hoc exemplar ubi p(ro) lubito usus fuerit ad eum transmittas, ego enim cu(m) ignore $(m)$ ubi $i a(m)$ agat, ${ }^{11}$ ad eum nihil iam litterarum dedi, ideo maxime q(uia) dubito num iter i(n) Italiam susceperit tum et(iam) q(uo)d nil aliud iam occurrat q(uo)d ad eum scribam. Vale.»

Cette note est donc une lettre de Florentius à Torrentius qui s'achève sur la formule classique de congé «vale»; elle occupe, avec le texte de CIL 6.2294, le premier des trois bifeuillets $(42 \mathrm{~cm} \times 25,5 \mathrm{~cm})$ qui forment l'ensemble du petit recueil MS 4350; ils présentent les traces d'une même double pliure dans le sens de la longueur et d'une pliure dans le sens de la largeur qui indiquent clairement que les trois bifeuillets furent envoyés ensemble à Torrentius, sous la forme d'une lettre. ${ }^{12}$

En même temps que la lettre, que je qualifierai de «lettre épigraphique,» en raison de son contenu, Florentius envoie donc une autre copie autographe de CIL 6.2294 destinée cette fois à Pighius; on peut vraisemblablement l'identifier avec le petit feuillet incorporé aujourd'hui dans le recueil de Berlin (f. 126v) dont Mommsen ignorait alors l'auteur:

Stockholm, " Acta Instituti Romani Regni Suecide 22 (1962) et Wolfgang Harms, «Eine Kombinatorik unterschiedlicher Grade des Faktischen: Erweiterungen des emblematischen Bedeutungspotentials bei dem Archäologen Jean Jacques Boissard,» in Mimesis und Simulation, ed. Andreas Kablitz und Gerhard Neumann, (Freiburg i. B.: Rombach, 1998).

(11) agat: ante corr.: agatur.

(12) Sont blancs les ff. 1v, 2v, 3r, 4v, 5r-v. 
"Berol. f.127 in scheda agglutinata manu aliena scripta. ${ }^{13}$ Le feuillet porte la même note que la copie du MS 4350, mais avec quelques variantes, comme l'omission du nom de l'évêque ("Monsignor del Giglio») qui est qualifié d'episcopus: "Fragmentum tabulae marmoreae longum uncias 20 latum uncias 6 , quod effossum est ante quatuor annos dum fundamenta splendidissimi aedificij sui [cavaret] sub monte Trinitatis (sive colle Hortulorum) episcopus.» Comme dans le MS 4350, une "lettre épigraphique» suit également le texte de l'inscription avec les mêmes renseignements que dans celle conservée dans le recueil de Torrentius: "fragmentum.......particulas fastorum civilium mensis Iulij, Augusti et Septembris ut colligere facile est ex tabula marmorea.... ... quae nunc extat apud clariss. (imi) D. (omini Achillem Maffeium et aliis D.(omini) Colloti.... ... impressam extare et brevi...Antonio Lanfredio... a quo hoc... fragmentum in quibusdam differt ut ex collatione app...."

Smetius reçut également une copie de CIL 6.2294, sans doute de la part de Pighius qui avait consenti à l'aider à reconstruire son corpus qui avait péri dans l'incendie de sa demeure le 13 janvier 1558. On trouve, de fait, un petit feuillet sur lequel Smetius recopie le texte épigraphique avant de l'intercaler entre les pages de son recueil de Leyde («in Lugdunensi in folio postea adglutinata") déjà formé à cette date. Lipse publiera le petit feuillet volant, en l'intégrant au reste du corpus de Smetius, avec un respect scrupuleux du manuscrit préparatoire. Finalement, Gruterus transmettra l'inscription à deux reprises dans son corpus, en citant tantôt Smetius comme sa source (236.9), tantôt Florentius à travers Lipse («a Florentio Lipsius», 236.4).14

\subsubsection{CIL 6.1690, 1691, 1692}

Les trois inscriptions suivantes (ff. $3 \mathrm{v}-4 \mathrm{r}$ ) furent copiées par Florentius au moment de leur découverte en 1561, dans une vigne sur le mont Célius: "Basis marmorea effossa in vinea s.(anc)ti Stephani rotundi an.(n)o 1561 in monte Caelio." Sans surprise, on trouve une copie de ces inscriptions, mais cette fois encore de la main de Pighius, dans son manuscrit de Berlin (f. 208); ${ }^{15}$ il y précise leur origine: "ex Nicolao Florentio." En marge de CIL 6.1690, à hauteur de la ligne 12, Pighius ajoute cette note: "Adhuc alia carmina 'Hic bis praefectus' in libro Marcelli papae.» Par l'intermédiaire de Pighius probablement, les inscriptions arrivèrent dans les mains de Smetius (69. 6-8) qui cite également leur auteur «a Nicolao Florentio accepimus.» Finalement, on les retrouve chez Gruterus (361.1) qui se fonde sur l'édition de Smetius ("a Smetio»), mais aussi sur la copie de Knibbius (361.2 et 363.2: «a Smetio et Knibbio») qui avait copié les trois textes dans son recueil de Berlin (f. 58).

(13) CIL 1.298. Il s'agit du f. 126v.

(14) Le seul autre témoin ancien, mais plus tardif, est une copie imparfaite de Girolamo Leandro junior (1574-1631) conservée dans le manuscrit que possédait Gaetano Marini et qui est aujourd'hui à la bibliothèque apostolique Vaticane (MS Vat. Lat. 9135, f.254). Il ne semble pas toutefois dériver directement de la pierre.

(15) On retrouve la lettre « $G »$ caractéristique dans la copie des textes épigraphiques. 


\section{CIL 6.1693}

La dernière inscription du MS 4350 fut découverte en même temps que les trois textes précédents: "Basis marmorea effossa Romae in vinea s. (ancti) Stephani Rotundi in monte Caelio.» Dans une note rédigée cette fois en italien, Florentius y précise les circonstances de la découverte et décrit les figures latérales à peine esquissées sur son dessin (Fig. 3a): "Nel horto di s(anto) Stephano rotundo al lato alla chiesa di santo Erasmo; dove sono state cavate le reliquie del atrio di Proculo viro clarissimo nel cui atrio nella colonna del peristilio erano instrumenti de i tre contrati scritti in rame fatti con soldati delle colonie Aelie Hadriane.» L'inscription, qui parvint à Pighius, sans doute à travers Torrentius, en même temps que les trois autres, figure au même endroit de son manuscrit (f. 208), avec l'indication de la date de découverte: «basis marmorea effossa in Caelio...s. Steph. Rotundi ann. 1561.» Elle est également présente au même endroit du manuscrit de Smetius (69.5: "A Florentio"), dans la catégorie des "Statuarum bases tabulaeque honorar.(ium).» Plus tard, un autre belge, Philippe van Winghe, arrivé à Rome en 1589 copiera l'inscription dans son recueil épigraphique conservé à Bruxelles (MS 17872-73, f. 15b) directement d'après la pierre (Fig. 3b), qui se trouvait à cette date dans une collection privée: "in basi ante aedes card.(inalis) olim Maffei.» ${ }^{16}$

\section{LE MS 4347-49}

Avant de retracer l'histoire et la diffusion des autres inscriptions que Florentius envoya à Torrentius au lendemain de leur découverte à Rome et qui sont conservées dans le MS 4347-49, voyons brièvement le contenu du recueil que Mommsen avait divisé en quatre parties de manière sommaire. ${ }^{17}$ Dans le coin supérieur gauche du f. $1 \mathrm{r}$, une note rédigée par une main que l'on peut maintenant attribuer à Florentius, indique que le deuxième évêque d'Anvers était bien le destinataire du recueil: "R.(everendissi)mo $D$.(omino) Laevino Torrentio."

La première partie, rédigée par Florentius, décrit quelques-unes des plus prestigieuses collections d'inscriptions de l'époque. Elle débute par un groupe d'environ deux cents inscriptions (ff. 1-5, 7-9, 11, 12, 13r, 14-19, 20v, 21, 22) conservées dans la vigne du cardinal Rodolfo Pio da Carpi ("In vinea rev.(erendissi)mi cardinalis Carpensis, f. $13 v »),{ }^{18}$ suivi d'une

(16) Sur Winghe, on consultera le très bel et riche article de Godelieve Denhaene, «Un témoignage de l'intérêt des humanistes flamands pour les gravures italiennes: une lettre de Phillipe van Winghe à Abraham Ortelius, » Bulletin de l'institut historique belge de Rome 62 (1992): 69-137.

(17) Ces quatre parties ne correspondent plus exactement à la numérotation actuelle. La description manuscrite de Mommsen se trouve dans les archives du CIL à Berlin (Akademie der Wissenschaften). Sont blancs les feuillets $2 \mathrm{v}, 3 \mathrm{r}, 4 \mathrm{v}, 5 \mathrm{r}, 6 \mathrm{v}, 7 \mathrm{v}, 8 \mathrm{r}, 9 \mathrm{r}, 10 \mathrm{v}, 11 \mathrm{v}, 12 \mathrm{v}, 13 \mathrm{r}, 14 \mathrm{r}, 15 \mathrm{r}, 16 \mathrm{r}, 17 \mathrm{v}, 18 \mathrm{v}, 19 \mathrm{v}, 20 \mathrm{v}, 21 \mathrm{r}, 22 \mathrm{r}, 23 \mathrm{r}, 24,25 \mathrm{r}$, $26 \mathrm{v}, 27 \mathrm{v}, 28 \mathrm{v}, 29 \mathrm{r}, 30 \mathrm{r}, 31 \mathrm{r}, 32 \mathrm{v}, 33 \mathrm{v}, 34 \mathrm{r}, 35 \mathrm{v}, 36 \mathrm{v}, 37 \mathrm{v}, 38 \mathrm{v}, 39 \mathrm{r}, 40,41 \mathrm{r}, 42 \mathrm{v}, 44 \mathrm{v}, 45 \mathrm{v}, 46 \mathrm{r}, 47 \mathrm{v}, 48 \mathrm{v}$.

(18) ff.1v-5v; ff.7r-9v, 11r-12v; 14v-22v; f.37r. Sur la collection d'inscriptions de Carpi, je me permets de renvoyer à mon article "Pirro Ligorio e le false iscrizioni della collezione di antichità del cardinale Rodolfo Pio di Carpi,» in Alberto III e Rodolfo Pio da Carpi collezionisti e mecenati (Atti del seminario internazionale di studi, 


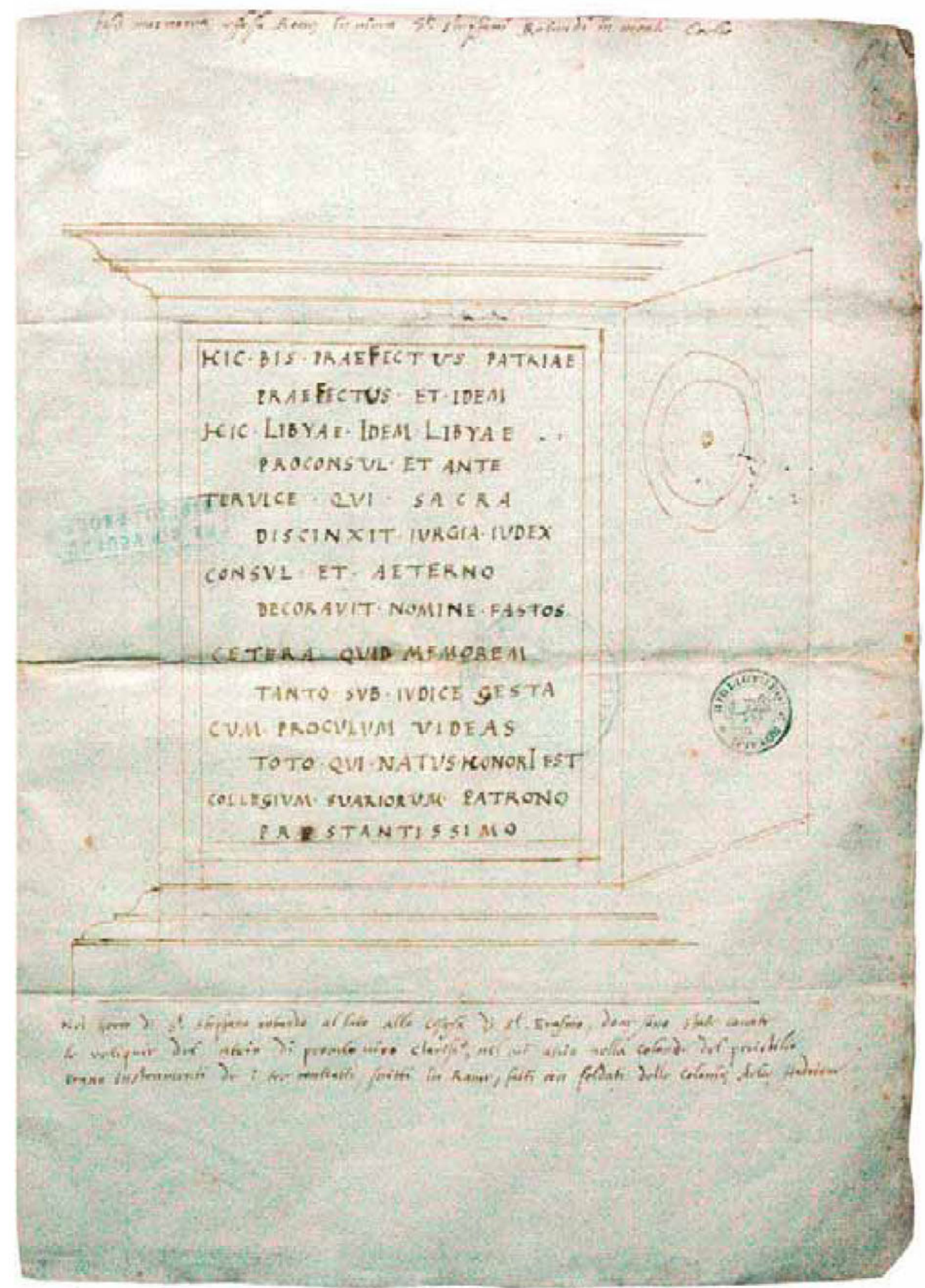

Fig. 3a.: CIL VI 1693 copiée par Florentius. 


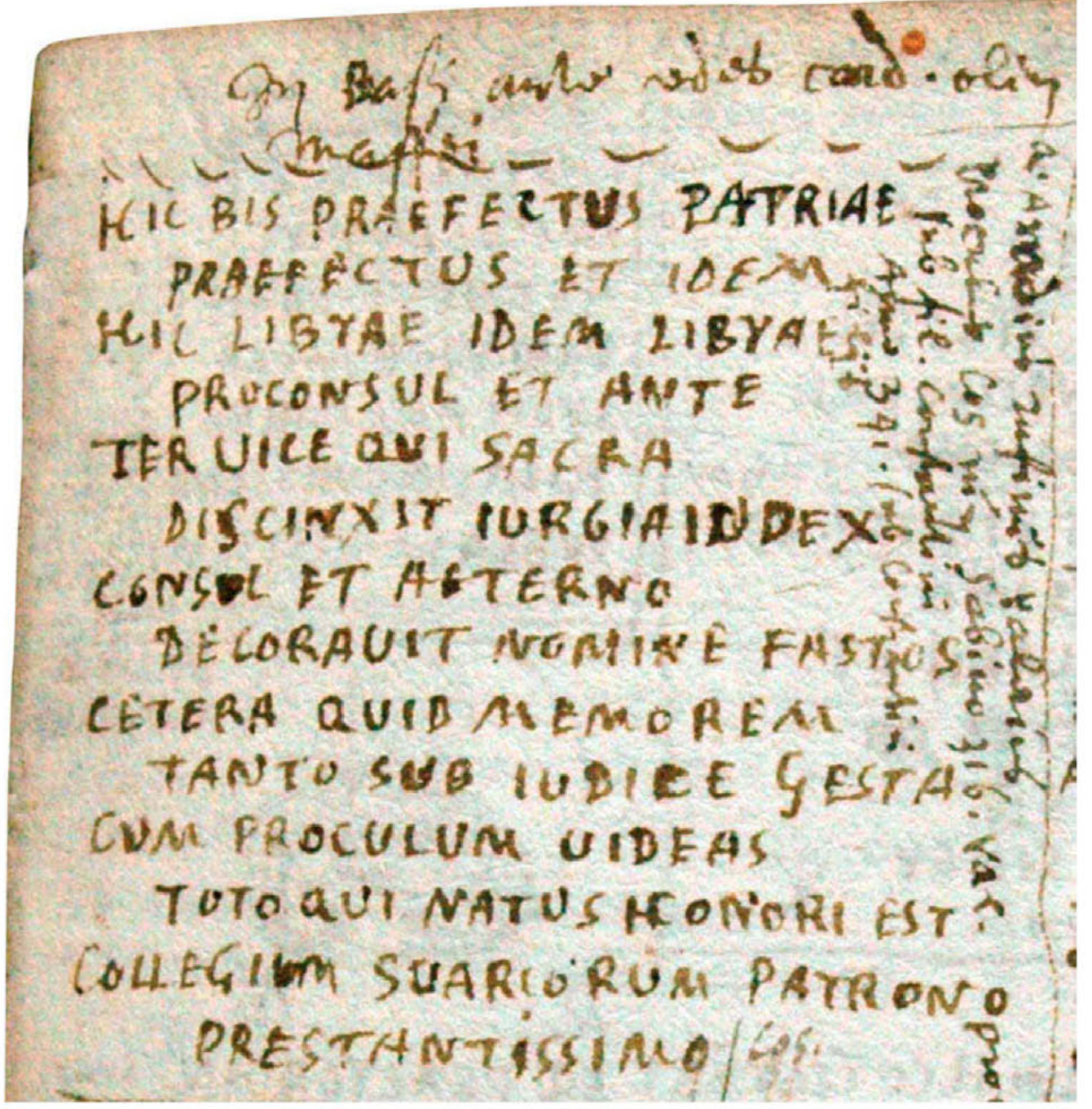

Fig. 3b: CIL VI 1693 copiée par Winghius. 
centaine de textes épigraphiques (ff. 23-33) de la famille Delfini ("Romae in aedibus Delphiniorum Marii p.ris et Attilii»: f. 29v); ${ }^{19}$ de seize inscriptions (f. $13 \mathrm{v}$ et f. 20r) qui formait la petite collection personnelle du père servite Ottavio Pantagato de Brescia ("Omnia infra scripta sunt ante cubiculum doctissimi patris Octavij Pantagati, f. $20 \mathrm{r}) ;{ }^{20}$ de quelques inscriptions, dont une grecque (f. 36r) de la collection de Bruto della Valle ( in aedibus Bruti della Valle, ff. 37v-39»); d'une inscription vue "In monasterio s.(anc)ti Pauli extra urbem» (f. 37r) et d'une autre conservée "intra monasterium s. (anc)tae Mariae in Via Lata" (f. 13v).

Le feuillet 35, que Mommsen attribue à la première partie du recueil, est en réalité un feuillet isolé inséré au reste du cahier qui ne semble pas rédigé par Florentius; il présente au recto le texte d'une inscription (CIL $6^{* * *}$ ) conservée "Romae apud cardinalem s. (anc)ti Angeli» et qui est également un fragment de Fastes.

La deuxième partie est constituée d'un bifeuillet étranger au cahier (ff. 43-44) qui présente les deux faces d'une inscription de Narbone (CIL 13.4333). Elle est rédigée par une «manus ignota» qui s'est fondée sur une copie diffusée par l'humaniste milanais Francesco Ciceri (1521-96) en 1567, comme l'indique la note qui accompagne le texte: "Questa memoria è stata ritrovata in Narbona nelle ruine della muraglia vecchia presso la porta regia. Copiata d'un marmo quadro, et la prima parte d'ung (sic) lato, la seconda dall'altro. Nella p.(rim)a riga de la seconda parte pare essere errore del scrittore NARBONESIS. Et nella 22 EADEM. Franciscus Cirerius A. Paulii fil. A 3 de genaro 1567.» Il n'est pas facile d'établir un lien entre cette copie d'inscription et Florentius même s'il semble qu'un lien existe, comme on verra plus loin. Lipse publiera l'inscription dans son Auctarium (p. 18) puis Pighius la transmettra à son tour dans son manuscrit (f. 191) mais en se fondant sur la copie conservée dans les "adversaria" de Lipse (Lips. 22, f. 2), comme l'indique la note en tête du feuillet: "Ex Lipsis adversariis."

La troisième partie (ff. 45r-48r), rédigée par une "manus ignota," concerne la numismatique. Elle est composée d'un feuillet isolé (f. 45) portant le titre: «addita ab (A. Schotto) Antonio Augustino arch.(iepiscopo)," ${ }^{22}$ suivi de la description iconographique de quelquesunes des divinités apparaissant sur les médailles: Sol, Cupido, Vulcanus, Vertumnus, Pomona,

Carpi, 22 e 23 novembre 2002), ed. Manuela Rossi (Tavagnacco: Arti Grafiche Friulane, 2004), 109-21. Je précise en outre que Heikki Solin prépare le catalogue complet de cette collection dont il a déjà publié quelques informations dans la préface de son édition des inscriptions du musée archéologique de Naples : «La collezione epigrafica Farnese tra Roma e Napoli,» in Catalogo delle iscrizioni latine del Museo Nazionale di Napoli, I. Roma e Latium, ed. Giuseppe Camodeca e Heikki Solin (Napoli: Loffredo, 2000), 11-44.

(19) Silvia Orlandi a recensé environ 150 inscriptions de la collection Delfini dans sa contribution : «Un contributo alla storia del collezionismo. La raccolta epigrafica Delfini,» Opuscula epigraphica 4 (1993).

(20) J'achève une étude sur Ottavio Pantagato et l'épigraphie qui sortira sur le prochain numéro de la revue Epigraphica.

(21) Richard Schofield, «Gioanni da Tolentino goes to Rome: A description of the Antiquities of Rome in 1490, Journal of the Warburg and Courtauld Institutes 43 (1980): 246-56.

(22) Schotto est barré. 
Harpocrates, Genius. Harpocrate, par exemple, y est décrit comme un: "Iuuenis cum cornucopia manu sinistra; dextro lato indice ori...quo silentium imperat. Lapis est lazuri. Meminit Ausonius explicat Angelus Politianus Miscellan. Cap. LXXXIII et an.....Goropius Becanus in Hieroglyphicis tom. VI." Au bas du feuillet apparaît la mention du sixième dialogue (DIALOGO VI) de l'œuvre homonyme de l'évêque espagnol Antonio Agustín (1516-86) cité dans le titre du feuillet. Andreas Schottus (1552-1629), également cité au même endroit, avait envoyé à Torrentius un exemplaire de l'œuvre du prélat espagnol. ${ }^{23}$ Le bifeuillet suivant, étranger au reste du cahier (ff. 46-48) est une planche de gravures de médailles grecques d'Alexandre, Philippe de Macédoine et Lysimaque; en son centre est collé un feuillet volant avec la gravure d'une médaille de Néron. Torrentius, passionné de numismatique, ${ }^{24}$ partageait cette passion avec Schottus et Agustín qu'il interroge, précisément à propos de ses Dialogos, dans une lettre du 30 août 1588. À cette date, Torrentius acquiert aussi l'édition de Smetius qui venait de sortir de presses de Plantin. ${ }^{25}$

La quatrième partie est un recueil acéphale d'inscriptions numéroté à l'encre en chiffres romains, du f. XXI (f. 49r) jusqu'au f. CIX (f. 93r); la numérotation au crayon, qui débute au premier feuillet du MS 4347-49, se poursuit donc au feuillet suivant (f. 94r) jusqu'au f. 176v. Ce recueil d'inscriptions incomplet, rédigé par une "manus ignota» dépend, selon Mommsen, de la sylloge de Pighius, conservée à Berlin: «Archetypum formae quadratae olim bibliothecae Lugdunensis Luzaci, nunc servatur Berolini in bibliotheca regia lib. pictur. A 61h. Exemplum esse ipsum a Pighio missum Grutero huius ostendit nota in f.1 adscripta haec: Jani Gruteri ex dono auctoris, cui antidorum misi aureos quatuordecim cum volumine mearum insctiptionum.' Alterum est in codic Bruxell. 4347. ${ }^{26}$ Dans l'attente de conduire une étude approfondie sur cette dernière partie du MS 4347-49, je voudrais simplement confirmer ici, par la comparaison des deux recueils, que c'est bien le copiste du manuscrit de Bruxelles qui a copié l'exemplaire de Berlin et non l'inverse (Fig. 4a et Fig. 4b); en effet, le copiste de Bruxelles omet de signaler des détails matériels comme la fracture de la pierre dans la quatrième inscription; il n'indique pas non plus le signe de renvoi au-dessus du " $V$ » de VERCELLESIS à la ligne 4 de la première inscription, de même qu'il ne souligne pas en pointillés les lettres liées de la cinquième inscription, au début de la ligne 3. Il semble aussi qu'un calcul trop juste de l'espace destiné aux commentaires marginaux l'a forcé à déplacer

(23) Agustin, Antonio. Dialogos de medallas, inscriciones y otras antiguedades. Tarracona, 1575.

(24) On consultera l'article de François de Callatay,, «Lambert Lombard (1505/1506-1566) et les monnaies antiques" dans Actes du colloque Lambert Lombard, Liège mai 2006 (à paraître).

(25) Jeanine De Landtsheer, «Torrentius literaire activiteiten in het prinsbisdom en in de Nederlanden, » in Laevinus Torrentius tweede bisschop van Antwerpen (Antwerpen: Kathedrale Kerkfabriek van Onze-Lieve-Vrouw, 1995), 31. L'existence d'une traduction latine de l'œuvre d'Agustin due à Schottus, qui vit le jour à Anvers en 1617, explique sans doute la confusion dans le titre, où le nom du jésuite indiqué initialement, est ensuite barré d'un trait et remplacé par celui d'Agustin. Si cette hypothèse est correcte, elle impliquerait aussi une intervention postérieure à la mort de Torrentius (1595) dans la formation du ms. 4347-4349.

(26) CIL 6:50 s.v. Stephanus Winandus Pighius. 


\section{DEORVM}

\section{$\overline{x L I X}$.}

MTRIKVAR

PSerititis?

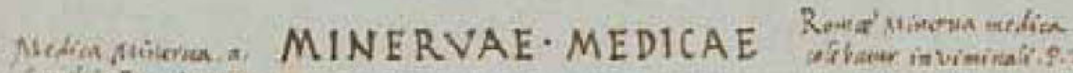

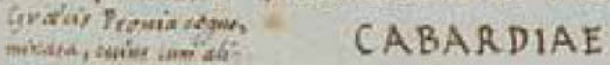

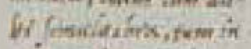

privis in inim tart

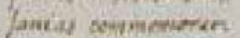

VALERIA. SAMMONIA

VैERCELLESIS.V.S.L.M

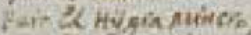

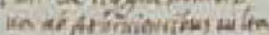

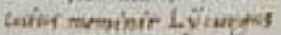

frikns.

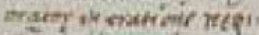

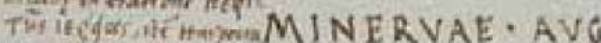

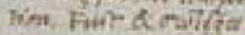

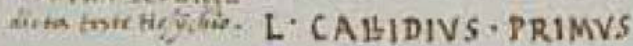

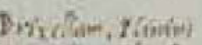

BRIXELLANVS - EX-ARG

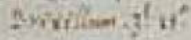

LIB. I1-1TEM-L'CALLIDIVS

Aion ninma, di mh PRIMVS.AVRES-ARGENTEAS

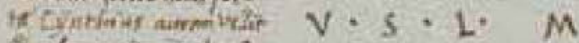

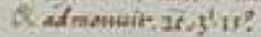

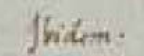

wits Amsene fir in, MINERVAE

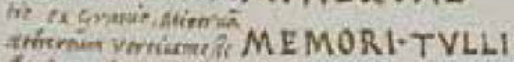

Q. Tementiati mivis

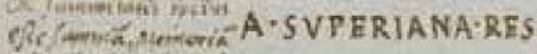

fore tordic wionvo RITVTIONE-FACTA

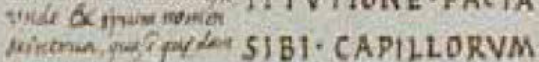

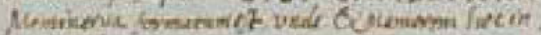

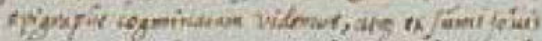

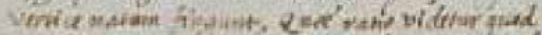

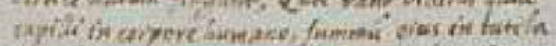

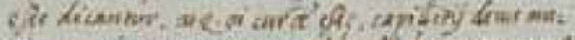

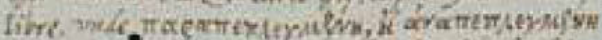

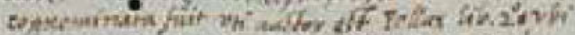

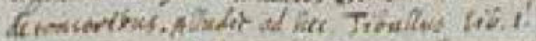

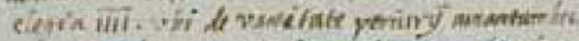

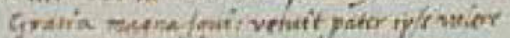

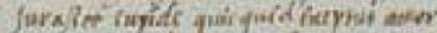

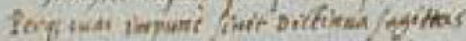

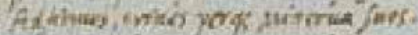

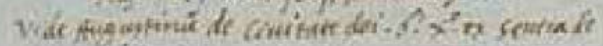

Fig. 4a: Inscriptions à Minerve.

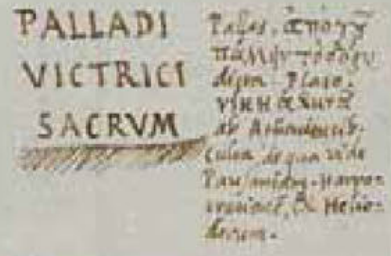

Banivenof

MINERVAE

AVG

M.AVISTIVS

HOMVNCIO

IIIII VIR-AVG

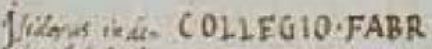

tinteribriation DONO-POSVIT

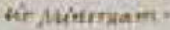

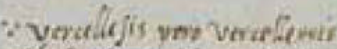

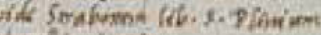
1. $17 \% / 3 y^{\prime} \cdot 4^{\prime}$ !

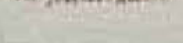


$\operatorname{tin} C R Y A L$

Placentic

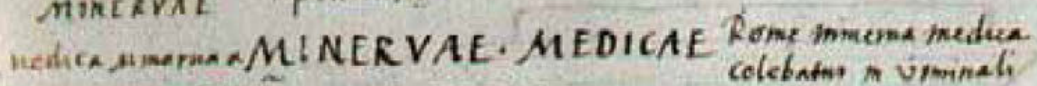

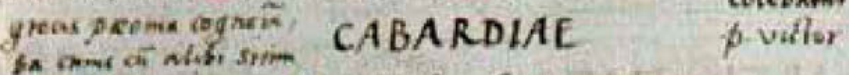

incm ThAmon VALERUA SAMMONIA

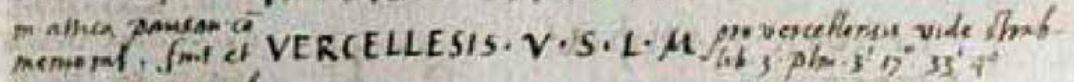

tygen, themens at

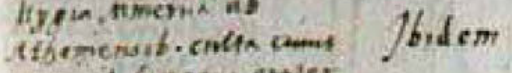

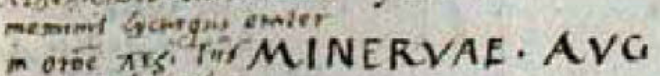

itstiar Jicm ber L CALIDIVS. PRIMVS

Gatigsa dista teite

sesuche

BRIXELLANVS-EXARO oth bDXiLe

PALLADLPathar íne

VICTRICI te dosividita.

SACRVMPhtovixa as gerra ab Alsem cosib. incton de LIG. II.ITEM-L-CALLIDIVS

the vide panim th

lates minemg of PRIMVS.AVRES-ARGENTEAS mement 6 ns ind

poete cowbiacs anrin $V \cdot S \cdot L \cdot M$

velfid of ad thewiot 2 ; is

thidew.

MINERVAE

vode Anobis th MINERVAE

A VG

M-AVISTIVS

or g,ame simeria MEMORJ.TVLII

ciberen verine et

is singmiase ysims A SYPERIANARIS

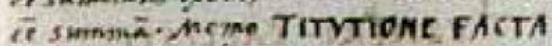

nö ados nemulh SIBI-CAPILLORVM

HONIVNCIO

liiilviR.AVG

COHFGIO FABR SISTm

Done. POStit $m$ hecme

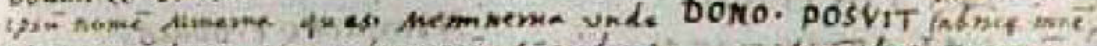

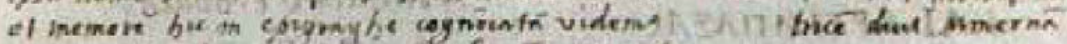

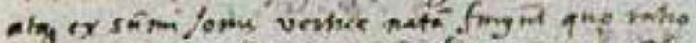

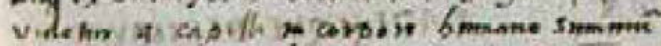

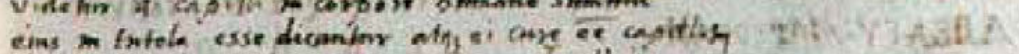

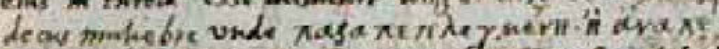

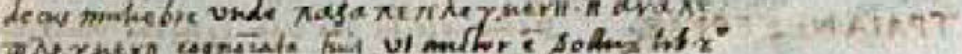

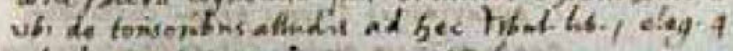

vbi de vawiate gerionin amome bee

grana maym ton vahis af io ve valew Jhrosst cosule gmequed methes amer

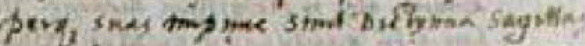

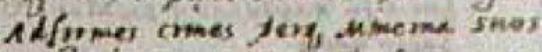

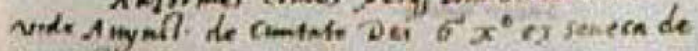
supentilions

Fig. 4b: Inscriptions à Minerve. 
à gauche le premier des deux commentaires relatifs à la deuxième inscription ("Brixellum...») ainsi que le commentaire de la cinquième inscription ("Isidorus...»). En revanche, il va jusqu'à recopier la numérotation en chiffre romain de son modèle (ici f. XLIX).

\section{CIL 6.1764}

La première des inscriptions récemment découvertes à Rome, qui apparaît dans le MS 4347-49, est rédigée sur un petit feuillet que Torrentius a inséré au sein d'un bifeuillet du groupe des inscriptions de la collection du cardinal di Carpi (f. 6r). Cela semble indiquer que Torrentius possédait déjà le recueil formé avant l'envoi de ces nouvelles inscriptions. Le texte épigraphique y est accompagné des précisions habituelles sur les mesures de la pierre, la date et le lieu de découverte: "Marmorea basis alta pedes 6. Effossa Romae in vinculo quo itur a spoglia Christi in columnam Traiani, sub aedibus Angelicae viduae Pauli Sceronis die octava Augusti an.(n)o 1564.” Une seconde fiche autographe est parvenue à Pighius, sans doute par le truchement de Torrentius (f. 126v), avec un commentaire presque identique: "Marmorea basis alta pedes 6. Effossa Romae in vinculo q.(uod) ducit spoglia Christi in columnam Traiani, sub aedibus Angelicae viduae Pauli Sceronis die octava Augusti an.(n)o 1564." On retrouve finalement la transcription de la même fiche dans l'édition de Smetius (69.14) qui précise que Florentius l'a envoyée deux mois seulement après sa découverte: «In basi marmorea alta pedes VI effossa Romae anno 1564 in viculo quo itur a Spoglia Christi in columnam Traiani, sub aedib.(us) Angelicae, vidude Pauli Sceronis. Et hoc ad nos transmisit Nicolaum Florentium anno s. s. sub mensem octobrem.» Gruterus, selon son habitude, publiera le texte dans son corpus (465.8). La date de découverte de l'inscription fournie par Florentius à travers les trois copies citées plus haut nous permet également de dater les deux copies de l'inscription rédigées par Pirro Ligorio (1512-83) et collées dans l'un des deux recueils épigraphiques d'Onofrio Panvinio (1530-68) de la Bibliothèque Vaticane (Fig. 5): «scritta in una base di marmo trovata a questi giorni nelle opere delle rovine del foro Traiano: Vat. Lat. $6036 \mathrm{f}$. $57 \mathrm{r}$ et $93 \mathrm{v}$." Cette identification nous permet d'affirmer que Ligorio aussi était présent au moment de la découverte de la pierre dans le forum de Trajan le 8 août 1564.

\section{CIL 6.1006}

La deuxième inscription est également rédigée sur un petit bifeuillet volant inséré dans le cahier (f. 10v); elle fut découverte à Rome à la fin du mois de septembre de l'année 1562: "Basis alta paulo plus 7 pedibus effosa Romae ante dedes d.(omini) Alexandri columnae, ad finem mensis septembris anno 1562. Plin. lib.3.5.4 colonia Arelata Sextanorum.» Elle est également présente dans le recueil de Smetius (59.8) avec des indications similaires: «Eruta Romae, апnо MDLXII et a Nicolao Florentio ad nos transmissa.» Gruterus publie à son tour le texte en se fondant sur Smetius (257.6) tandis que Lipse, qui avait édité la copie que Smetius avait ajoutée à son corpus manuscrit, ne jugea pas nécessaire de la publier une seconde fois et se contenta de la copier à deux reprises dans ses «adversaria» (Fig.6, 


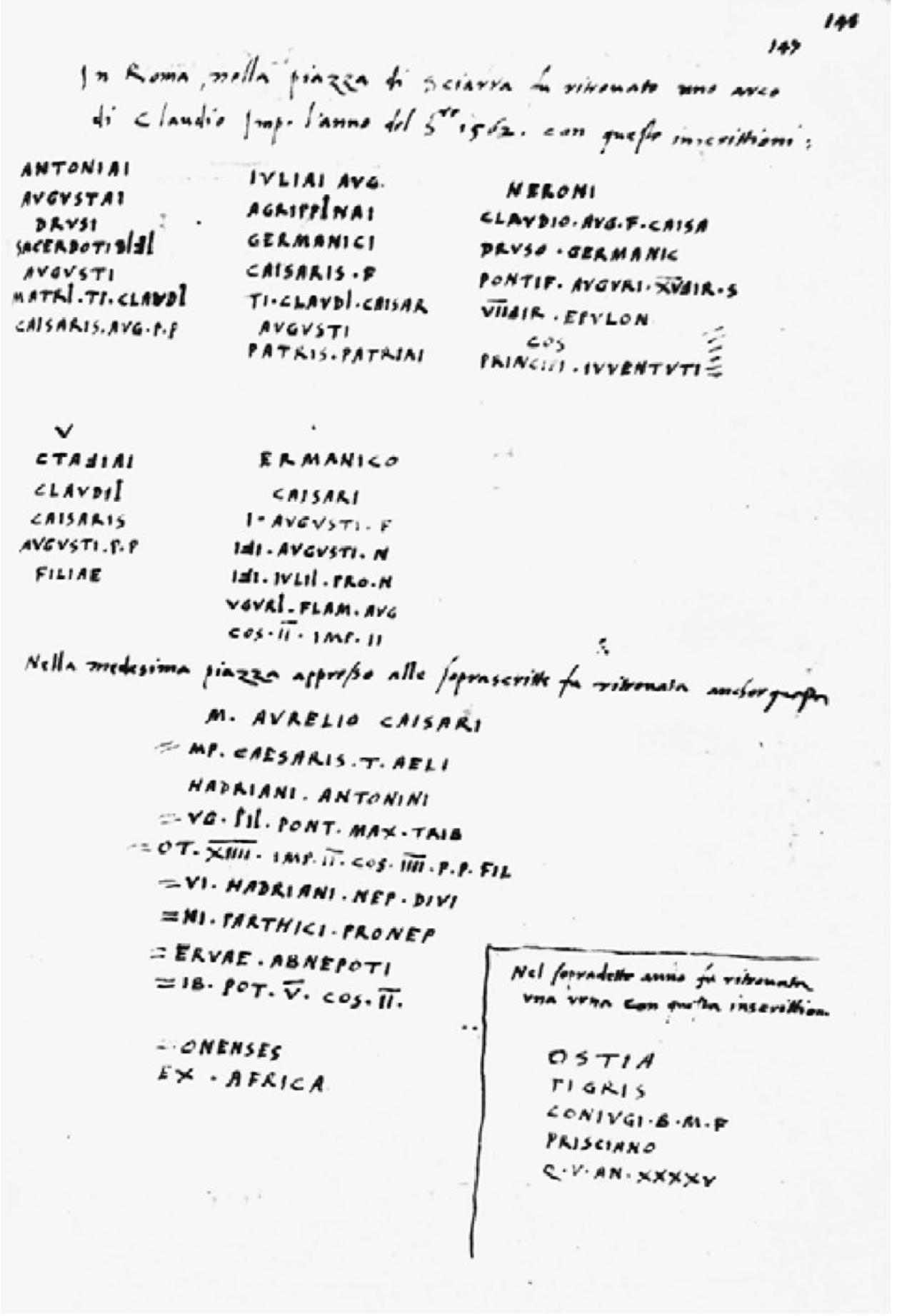

Fig. 5: CIL VI 1764 copiées par Pirro Ligorio. 


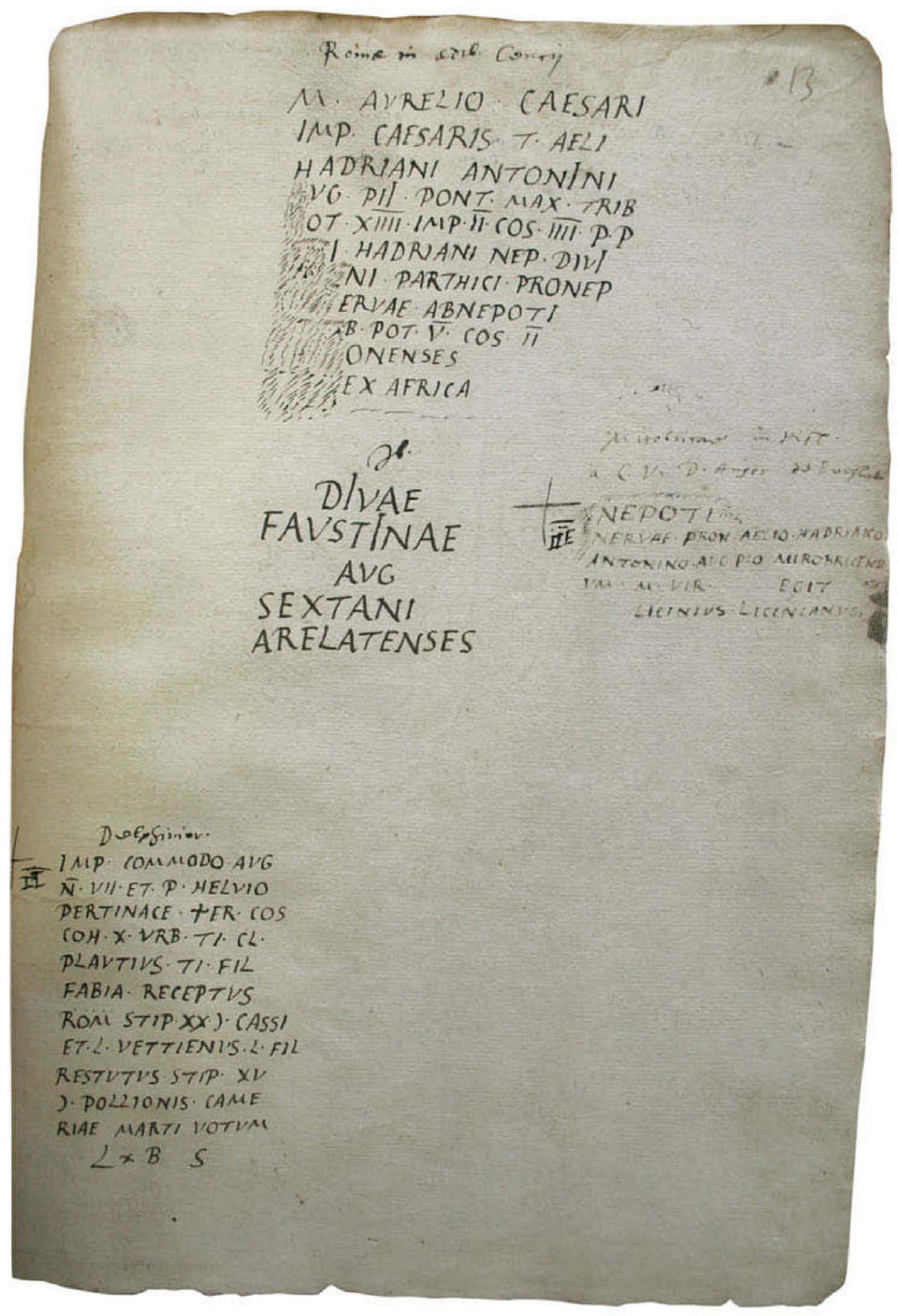

Fig. 6: CIL VI 1006 copiée par Lipse. 
ff. 13 et 14). La copie que Pighius nous transmet à son tour dans le recueil de Berlin (f. 196) vient d'une tradition indépendante, totalement inconnue par ailleurs, et italienne cette fois, puisqu'il précise qu'elle vient d'Orsini: «E Fulvi Ursini.» Pighius nous apprend qu'au moment où Orsini copie l'inscription, elle se trouvait déjà dans une collection privée romaine: "In aedibus Cesij mag.....»

\section{CIL 6.10211}

L'inscription suivante (f. 34v) fut découverte aux environs de 1561, près de l'arc de santo Vito, mais Florentius précise qu'au moment où il la copie, la pierre est dans la collection du cardinal Sant'Angelo (Fig. 7a): "Fragmentum tabellae marmorea repertum in vinea quadam Romae p.(ro)pe arcum s.(anc)ti Viti, circa anno 1561; altum plus minus tre palmos et tantundem latum. Q(uo)d nunc est in Musdeo R.(everendissi)mi cardinalis S. (anc)ti A[ngeli]." Selon le processus désormais prévisible, Torrentius fit parvenir à Pighius une autre copie autographe de Florentius (f. 116) qu'il colla dans son recueil (Fig. 7b). Smetius à son tour copia l'inscription reçue sur un feuillet avant de l'intercaler entre les ff. XII et XIII de son corpus déjà prêt pour la publication (Fig. 7c), accompagné des mêmes renseignements et du nom de Florentius: "Fragmentum tabulae marm.(orea) quadratum, altum plus minus palmos 3. Effossus in vinea quadam Romae prope arcum s. (anc)ti Viti, circa annum 1561. Quod nunc asservatur in Musaeo card.(inali) S. (anc)ti Angeli. Missum Roma per Nicolaum Florentium.» Lipse édita le texte (f. XII, 12), avec la fidélité déjà soulignée plus haut, en l'insérant au sein du recueil de Smetius déjà achevé (Fig. $7 \mathrm{~d}$ ).

\section{CIL 6.1186}

Au bas du même feuillet apparaît le dessin d'un monument en mauvais état précédé d'une indication (Fig. 7a): "Fragmentum baseos altum pedes 5 1/2 effossum Romae ante mensem aut alterum sub aedibus privatis prope sacellum s(anctae) Mariae Lorettae non procul a columnae Traiani imp." Sous le dessin est ajoutée une seconde note qui est la seconde "lettre épigraphique" de ce recueil: "Aliud vero fragme(n)tum triumphorum $q(u o) d$ ad te ante transmisi descriptum ex exe(m)plari. D(omini) Fulvij Ursini, postea contuli cum ipso marmore, repperiq(ue) rectissime descriptu(m) fuisse. Idq(ue) audio ex eodem Fulvio partem eius esse, quod ante aliquod [sic!] annos [1563] in foro romano effossum, inde in area Domus Capitoline Conservatoru(m), ab card(inale) Fern[esio] positum fuit. Hoc aute(m) praesens triumphorum ${ }^{27}$ fragmentum non ita procul a foro iuxta aedes sancti [Andreae] in Portugallo, in vinea D(omini) Ludowici Matthei, hoc an(no) circa mensem februarium effossum.» Torrentius, sans doute, fit parvenir à Pighius une copie autographe de Florentius (f. 116) accompagnée du même commentaire (Fig. 7b): "Fragmentum baseos altum pedes 5 1/2 effossum Romae ante mensem aut alterum sub aedibus privatis prope sacellum s(anctae) Maria Lorettae non procul a columnae Traiani imp.; s sous le texte, Pighius ajouta de sa main la date de réception de l'inscrip-

(27) triumphorum: add. supra lin. 


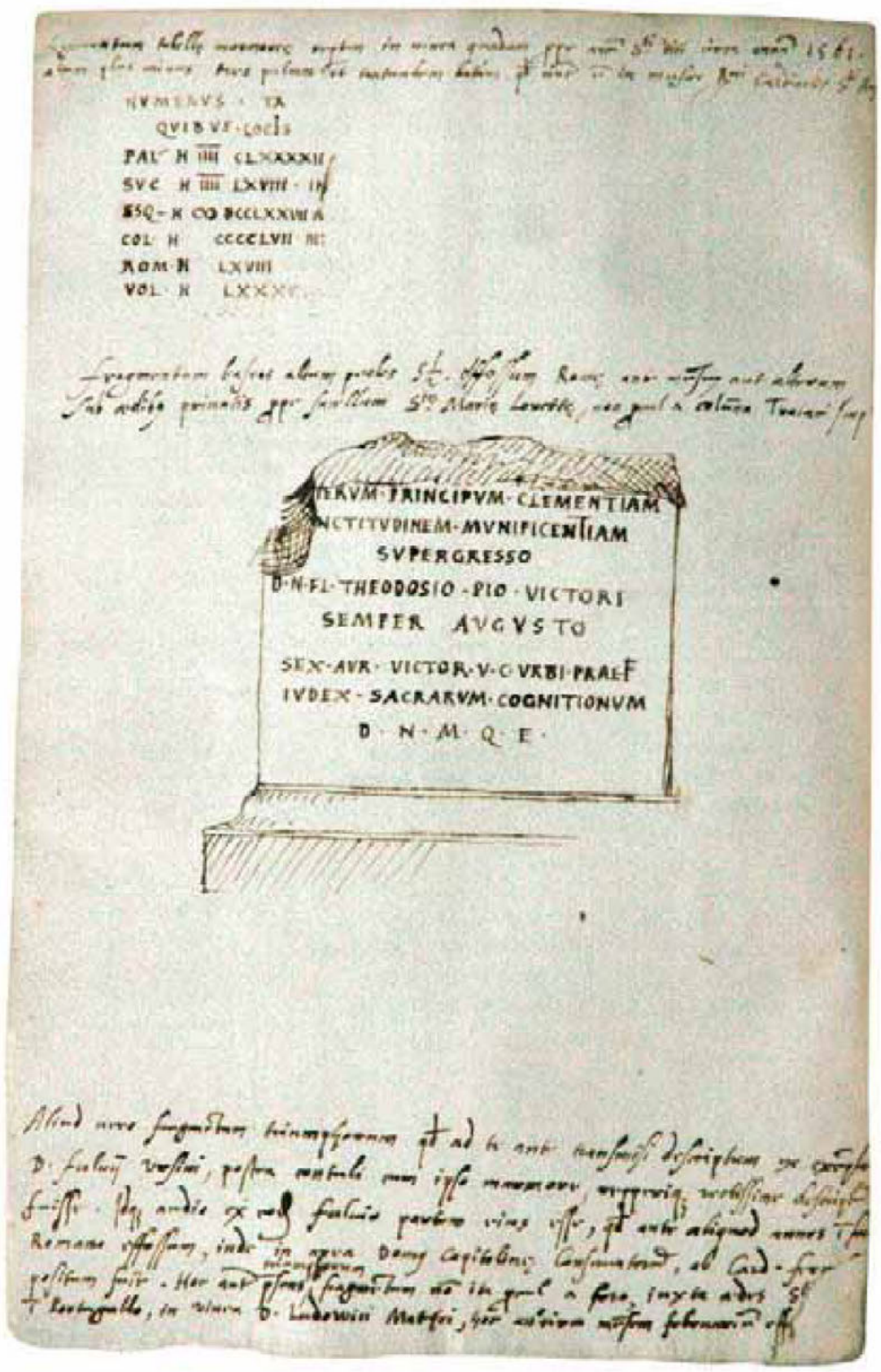

Fig. 7a: CIL VI 10211 copiée par Florentius. 


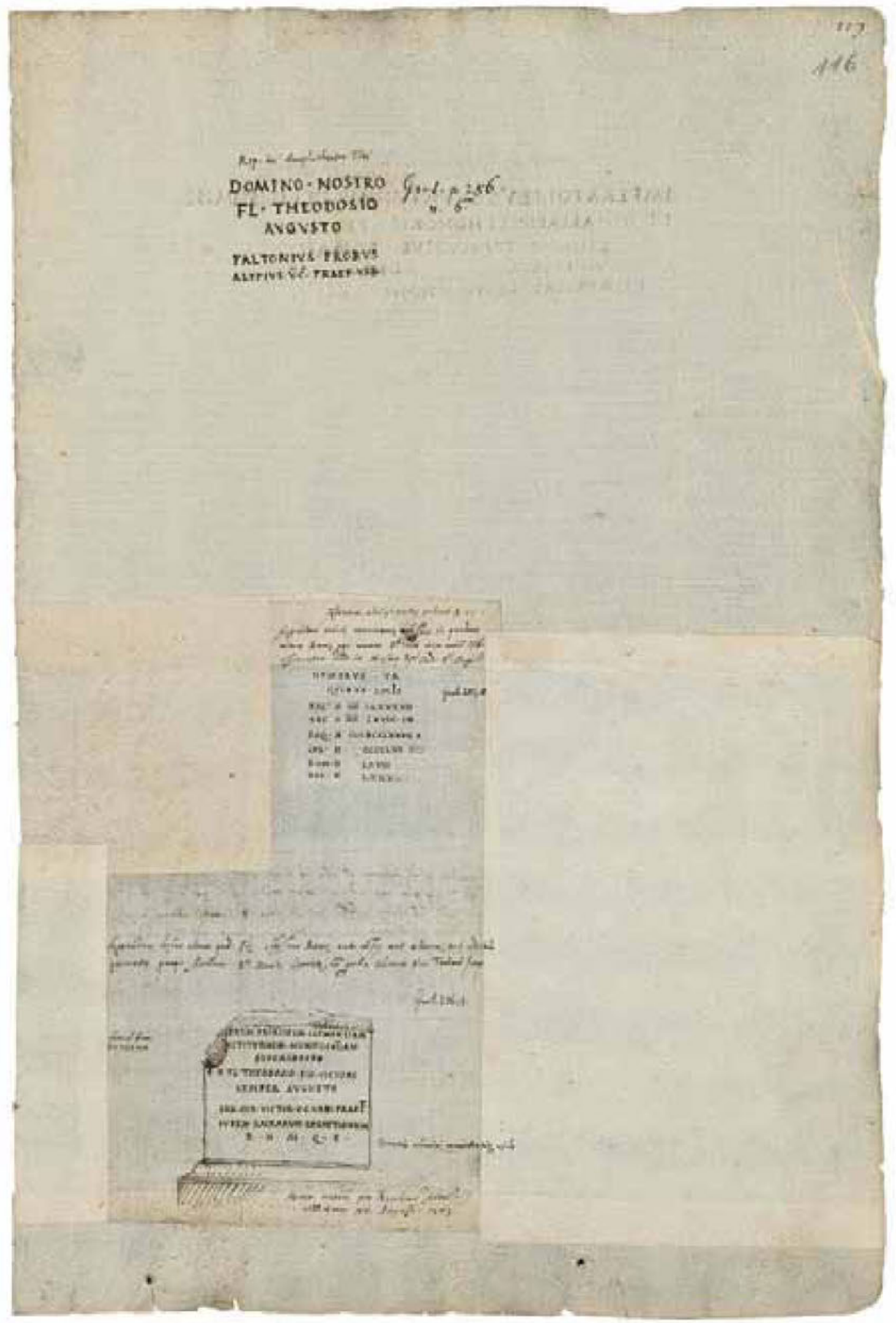

Fig. 7b: CIL VI 10211 copiée par Pighius. 


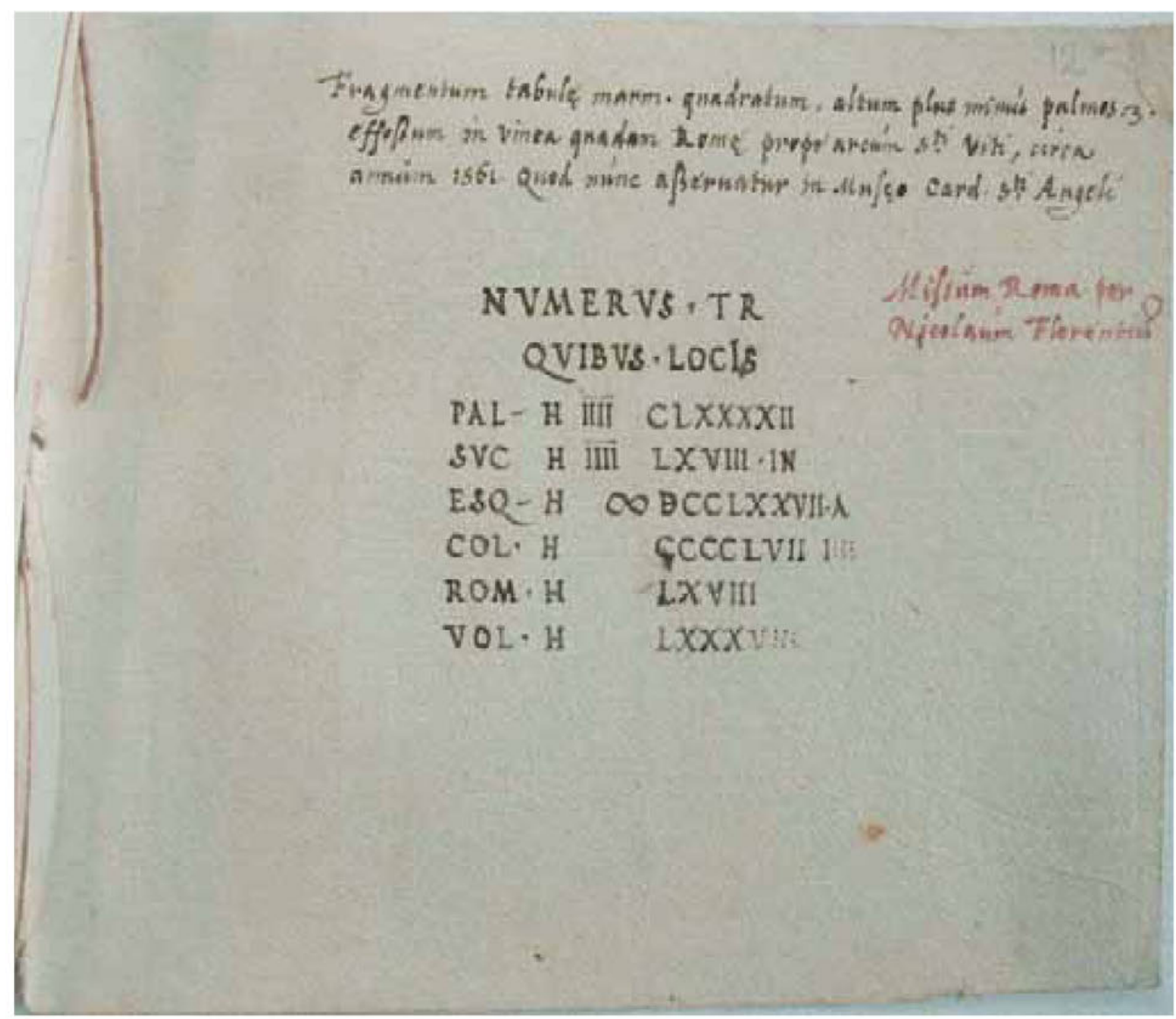

Fig. 7c: CIL VI 10211 copiée par Smetius.

tion: "Roma missum per Nicolaum Florentium, redd. Est mihi XII Augusti 1563." Au recto du feuillet reçu (f. 116v) apparaît une note contenant les mêmes renseignements que ceux de la "lettre épigraphique" à Torrentius: "Fragmentum aliud Fastorum quod olim ad te misi (?) quod in Capitolio ante aliquot annos positum est per (?) esse 1563 februarium effossum fuit in vinea d.(omini) Ludovisi Matthei quae est ad radices Esquiliniorum et idque postea (?) divi Andrea in Portugallo (?).» Smetius transmet l'inscription dans son corpus (f. 59, 10) en nous indiquant une fois de plus que Florentius l'envoya au lendemain même de sa découverte: "Fragmentum baseos altum pedes $51 / 2$ effossum Romae in foro Traiani haud procul a columnae eiusdem anno. 1563. Missum Roma per Nicolaum Florentium anno 1563." 
Fragmentum epistylï, effoffum ad circum Flaminium, vbi vulgo Le boreghe obfcure:

I2.

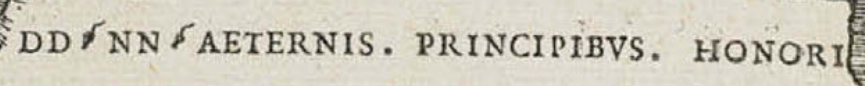

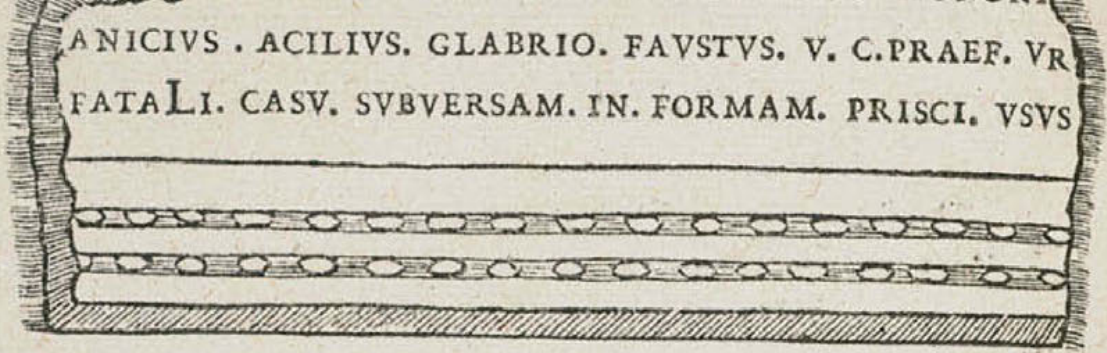

Fragmentum tabule marm.quadratum, altum plus minus palmos 3 . effoljum in vinea quadam Rome prope arcum $S^{t i}$ Viti, circa annum is 61 . Quod nunc afferuatur in Mufao Card. $S^{\text {ti }}$ Angeli

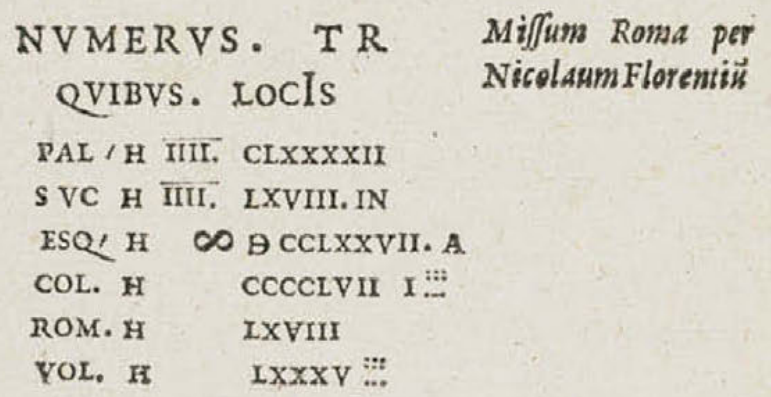

In duabus bafibus, in foro Rom. repertis, \&

In $S^{\text {ro }}$ Paulo, via Oftienfi. tertia, qua est in foro Traiani.
I4 FABIVS.TITIANVS V. C. CONSVL PRAEF. VRBI CVRAVIT

In $S^{\text {ro }}$ Vito,ad arcum Gallieni in macello Lisise in bofi.

II FL.EVRYCIES EPITYNCHANYS $\overline{V C}$ PR $\overline{A E F}$. $\overline{V R B}$. CONDI

In finistro latere

Fig. 7d: CIL VI 10211. 


\subsubsection{CIL 6.33-35}

Florentius précise la date et le lieu de découverte des trois inscriptions de dédicace à Apollon et Mercure (f. 41v): "Effossa hoc anno 1562 in vinea Tarquini Securi inter colles Aventinum et Testaceum." Il donne ensuite les mesures de la première pierre: "basis alta circiter pedes quatuor," suivies de celles de la suivante: "basis // magnitudinis." La troisième inscription (CIL 6.35) qu'entoure une légère esquisse d'autel, est accompagnée de la description des figures gravées sur les deux côtés: "ara quadrata. Cormus aut cornix ad arborem adstans capite erecto. Corona dependens ex arbore. In tergo hoc est adversa parte eiusdem arae videtur esse arcus Turcinus cuique extrema una pars in sequentis caput desinit, non altera eiusdem pars q. saxum ibidem ereptum sit, desinatur."

Pour une raison inconnue, ni Pighius, ni donc Smetius non plus n'eurent connaissance, semble-t-il, de ces trois inscriptions. Elles apparaissent, en revanche, dans les "Adversaria» de Lipse (f. 19) qui déclare avoir vu CIL 6.34 ("vidi»), au moment où elle se trouvait "in dedibus Maffeiorum." C'est là aussi que la virent les deux autres belges, témoins privilégiés de l'histoire de ces inscriptions: Winghius (f. 9: «in aedibus Maffeiorum») et Knibbius (f. 57). Tout comme Lipse, ils ont décrit de manière indépendante le texte d'après la pierre. Gruterus (36.7 et 54), qui n'avait pas trouvé de texte édité chez Smetius, se fondera une fois de plus sur l'exemplaire de Knibbius conservé à Berlin (f. 57).

\section{CIL 6.1316}

La dernière inscription semble bien rédigée par Florentius bien que l'écriture soit plus grande. Elle est accompagnée une fois de plus des mesures du fragment et de son lieu de découverte: "Marmor istud est fere altitudinis duoru.(m) pedum latitudinis vere plus trium et crassitudinis fere eiusdem. Liter(a)e superioris lineae sunt longae tres uncias inferioris vero lineae sunt fere et trium unciarum. Effossus est Romae prope arcu. (m) Sept.(imi) Severi sub i...." ${ }^{28} \mathrm{La}$ présence, à deux reprises, de l'inscription dans le recueil de Pighius (ff. 127 et 191) pourrait indiquer que Florentius est l'auteur de la copie conservée dans le MS 4347-49.

L'inscription eut aussi une transmission italienne, comme l'indique la copie du recueil d'Achilles Statius (Achille Estaço) conservée à la Biblioteca Vallicelliana (f. 72v et 75); Orsini aussi transmet le texte dans deux de ses ouvrages imprimés, mais sans préciser sa source. Il invoque ainsi le témoignage de CIL 6.1316 au moment de parler du temple Saturne dans son livre sur les Familiae romanae publié à Rome en 1575: "Saturni autem aedem de manubiis Plancum fecisse, alterius quoque inscriptionis fragmentum quod Romae prope ipsam Saturni aedem sueprioribus annis repertum esse. Eius exemplum subiecimus: L. PLANCUS L. F. COS. IMP. ITER DE MANIB; " ${ }^{29}$ on la retrouve, toujours au même sujet, dans ses Fragmenta historicorum, parus à Anvers chez Plantin en 1595: "Ex quo Saturni templum et aerarium ibi fuisse intelligere possumus L. PLANCUS etc. ${ }^{30}$

(28) Le feuillet est tronqué à cet endroit.

(29) Notae ad Suetonium ad cap. XXIX, p. 472.

(30) S.v. Munatia, p. 168. 


\section{LE MANUSCRIT DE PIGHIUS (MS LAT. FOL. 61)}

Deux fiches autographes de Florentius qui transmettent les inscriptions CIL 6.222 et CIL 6.1777 l'une en dessous de l'autre (Fig. 8), sont toutes deux conservées dans le recueil de Pighius (ff. 126 et 209); il se pourrait que Torrentius ait envoyé les deux exemplaires par inadvertance à Pighius, se privant ainsi d'une fiche à insérer dans son propre recueil, le MS 4347-49.

\section{CIL 6.222}

Dans les deux copies, l'inscription à Calpurnius Piso est précédée, à quelques détails près, des mêmes indications concernant les mesures, le lieu de découverte de l'inscription et celui de son actuelle conservation: "Tabula haec marmorea longa circa ped. 2 1/2 effossa ante (?) in vinea R.(everendissi)mi archiepiscopi de Maximis, in monte Aventino, nunc extat in atrio domus suae Romae anno 1570 die 29 octobris."

\section{CIL 6.1777}

L'inscription à Vettius Agorius Praetextatus est accompagnée dans les deux cas de l'indication de ses mesures: "basis haec alta est ped. 4 effososaque; ... sequitur tabula eodem tempore;» elle est également conservée dans le vestibule de la demeure "R.(everendissi)mi archiepiscopi de Maximis. "Sa présence dans la demeure du prélat, où Florentius la vit sans doute aussi le 20 octobre 1570, comme CIL 6.222, pourrait indiquer qu'elle fut découverte, comme cette inscription, dans la vigne de l'archevêque dei Massimi. C'est là que Winghe copiera lui-même les deux inscriptions ( 2 f. 6) à partir de 1589, quand il arrive à Rome.

\section{CONCLUSION}

Quoi qu'il en soit, ce double témoignage nous permet de découvrir que Florentius était encore en Rome en 1570 et qu'il avait accès aux plus brillantes demeures de l'époque. Il n'en reste pas moins que c'est entre 1561 et 1563 qu'il envoya le plus d'inscriptions à Torrentius avec requête de les communiquer à Pighius. L'examen des notes accompagnant ces inscriptions nous a montré que Pighius avait transmis à son tour ces mêmes textes à Smetius et l'on peut désormais ajouter le nom de Florentius aux amis qui ont permis à Smetius de reconstituer son corpus détruit, comme on l'a vu, dans l'incendie de sa demeure. Son ami Marcus Laurinus (Marc Lauweryn), seigneur de Watervliet, l'avait beaucoup soutenu après le drame et avait réussi à convaincre ses amis, Pighius, Antoine Morillon (c. 1520-56) et Carolus Clusius (Charles de l'Ecluse, 1526-1609) de mettre leurs fiches à la disposition de Smetius. Au début d'une lettre qu'il lui écrit quelques jours après l'incendie fatal (27 janvier 1558), Smetius remercie Pighius de sa générosité et expose sa méthode pour reconstituer son corpus: "Haud facile credas, doctissime Pighi, quantum mihi accesserit animi ad prosequendas illas inscriptiones, postquam apud te fuissem tuaque omnia vidissem, ac etiam 


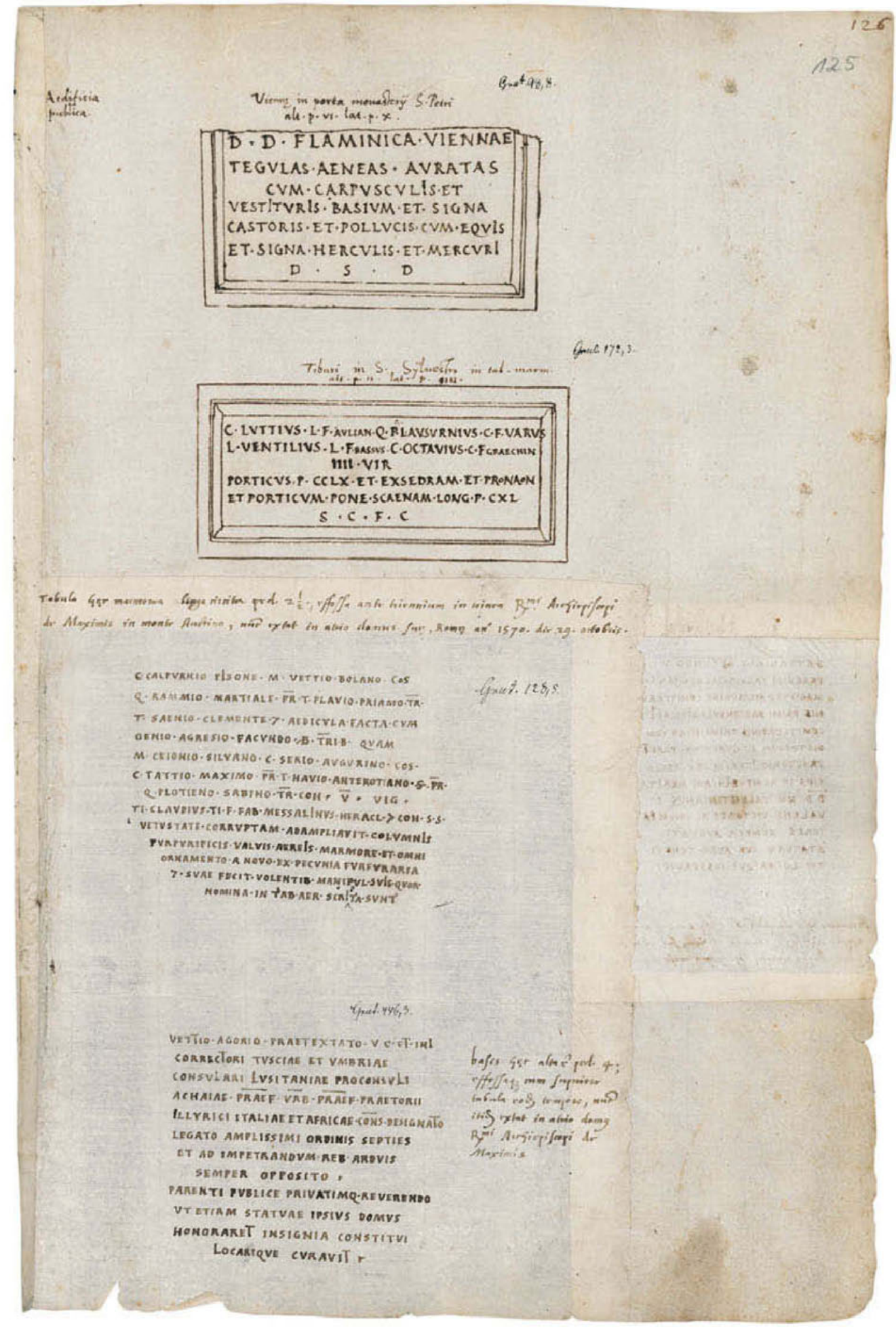

Fig. 8: CIL VI 222 et 1777 copiées par Florentius. 
acceptis a te multis egregiis epigraphis locupletior factus essem. Cum itaque domum redissem, eas suo quamque loco libro meo inserui; ac deinde schedas meas omnes diligentissime excussi, et quicquid in eis observatu dignum etiam quantulumcunque minutum reperi, eidem libro aut additis aut insertis foliis adiunxi. $n^{31}$

Florentius fit parvenir à Lipse des copies d'inscriptions romaines, mais aussi des textes venant d'autres régions d'Italie et d'Europe, comme l'inscription qui se trouvait à Rieti (p. 50): "in aedibus Petri Silverij» ("DIS MANIBVS/ C. IVLIO C. F. / VOLTINIA LONGINO DOMO») et qui lui vient: "A Nic.(olao) Flor.(entio)." C'est encore le cas d'un recueil d'inscriptions de Dalmatie, aujourd'hui perdu, qui fut, à un moment donné, entre les mains de Florentius et d'Orsini. Lipse, toujours dans son Auctarium, précise qu'il a reçu l'inscription de Spalato CIL III 1935 ( CONSENTIO DEORUM/ MARIAN. ASOZEMENAE/ IMPERIO FECIT") de Florentius ("a Nic.(olao) Flor.(entio); " quant à Gruterus, la deuxième source imprimée de l'inscription («CONSENTIO DEORUM/ MARIANUS SOZOMENUS./ IMPERIO...C.M. EX VOTO FE.»), il indiquait qu'elle lui venait d'Orsini ("ex ursinianis"). Ainsi, l'étude de l'Auctarium de Lipse nous permettra de reconstituer le corpus perdu des inscriptions de Spalato à travers les copies fidèles de Florentius que Mommsen qualifie, à cette occasion d' "homo probus." Il écrira à propos de CIL 3.1935: "titulus adhuc extans Spalati ibique, a Lipsio (i.e. a Florentio) recte refertur; nisi quod in lapide est Marcana Sozomene.»

De retour de son séjour romain, Lipse, toujours à l'affût de nouvelles inscriptions, demandera à son ami Orsini de lui faire parvenir, par le truchement de Florentius, toutes les récentes trouvailles: "In quo genere quoniam omnium iudicio facile princeps, non miraberis si hoc a te potissimum petam ut si quid a discessu meo inventum aut erutum est novi, quod legi nostrae interesse putabis, id ad me per N.(icolaum) Florentium transmittas. In quo incredibile dictu est quam gratum mihi facuturus sis. $)^{32}$ On peut ainsi fixer l'arrivée de la vingtaine d'inscriptions reçues de Florentius, que Lipse publiera dans son Auctarium, entre la date de son départ de Rome (1570) et celle de l'édition du corpus (1588).

L'examen des recueils de Torrentius m'a montré encore que Florentius prend toujours soin de préciser le lieu où les inscriptions sont conservées, la forme du support et ses mesures ("tabula alta ped. $\left.1 \frac{1}{2}, f .1 v »\right)$; il signale l'état de conservation de la pierre et indique les fractures notamment par un dessin sommaire ("fragmentum tabulae oblongae ped. 3, ibidem"). C'est aussi le cas quand il s'agit de figurer des détails iconographiques (f. 9v). Florentius indique aussi les particularités ou les anomalies qui se trouvent sur la pierre, comme la graphie MERENTI où la lettre «I» est superposée au «T; la graphie "ATHICTVS» ou encore l'abréviation "INFELICISSIM. »

Florentius est un témoin privilégié, des inscriptions découvertes à Rome au milieu du seizième siècle et parfois le seul témoin oculaire comme dans le cas de CIL 6.2294. Cependant, même dans les rares cas où l'on trouve, pour les inscriptions étudiées, une tradition

(31) La lettre est publiée par Mommsen dans le compte-rendu de la séance du 28 juin, Sitzungsberichte... (1866): 424 .

(32) La lettre est publiée par Pierre de Nolhac, La bibliothèque de Fulvio Orsini (Paris, 1887), 438. 
italienne, Florentius y est très étroitement lié, ce qui révèle, de manière encore imprécise, ses liens avec les érudits présents à Rome à cette époque. C'est ce que l'on constate si l'on revient un instant sur quelques-unes des inscriptions du MS 4347-49.

En septembre 1566, Ciceri envoya une copie de l'inscription de Narbone CIL 13.4333 à Aldo Manuzio junior (1547-97) qui se trouvait à Rome; l'érudit romain l'inséra dans l'un de ses recueils épigraphiques (MS Vat. Lat. 5234, f. 245); $;^{33}$ une seconde copie, vraisemblablement rédigée par un copiste, est conservée dans un autre de ses recueils (MS Vat. Lat. 6040, ff. 84-85); son contenu nous indique l'existence d'un lien avec la copie de Florentius du MS 4347-49: "Questa memoria è stata ritrovata nuovamente a Narbona ne le ruine de la muraglia vecchia presso la porta regia; per quanto sintende da una lettera data ali 3 di genaro 1567 ne la quale protesta colui che scrive d'haverla fedelmente copiata d'un marmo quadro e la prima parte d'un lato, la seconda da l'altro suspica pero che siano alcuni errori del medesimo scrittore come ne la prima riga de la seconda parte: NARBONESIS nella xxii EADEM quali non ho voluto mutare; ho ben preso ardire di mutare nella riga XVIII de la prima parte INCOLL et scrivere INCOLIS. AD. Franciscus Cicereius. Aldo Manutio Pauli F. D. D.» Dans une autre "lettre épigraphique» autographe du 31 décembre 1569, conservée dans un troisième recueil de Manuzio (MS Vat. Lat. 5237, f. 358r), Ciceri lui promet l'envoi d'autres textes découverts toujours à Narbone que lui a envoyés un certain Bartolomeo Aresio: «Mando la copia d'alcune inscrittioni notabili, di novo cavate dalle muraglie di Narbone, quali m'ha dato il gentil giovane et molto nobile, il s. (ignor) Bartolomeo Aresio." Il n'est pas impossible que Florentius ait reçu de Manuzio la copie de CIL 13.4333, comme semble l'indiquer en outre la présence, dans la copie du MS 4349 de la correction INCOLL en INCOLIS suggérée par Ciceri à Manuzio (MS Vat. Lat. 6040, ff. 84-85); Florentius aurait ensuite transmis la copie exécutée par un copiste à Torrentius, sans perdre de temps à recopier une inscription de soixante-neuf lignes.

Pour ce qui concerne CIL 6.921, le seul autre témoin qui nous livre ensemble les six fragments est conservé dans un autre receuil de Manuzio (MS Vat. Lat. 5237, f. 147). Mommsen précise qu’il s'agit de la copie d'une «manus ignota, » révélant ainsi la complexité de la transmission des textes épigraphiques et en particulier dans la seconde moitié du XVI ${ }^{\mathrm{e}}$ siècle, qui est la période la plus féconde de récolte d'inscriptions latines en Italie et en Europe. Le texte de CIL 6.921 y est précédée d'une indication en italien: "In Roma, nella piazza di Sciarra fu ritrovato uno arco di Claudio imp. L'anno del S.re con queste inscrittioni" et plus loin: "Nella medesima piazza appresso alla soprascritta fu ritrovata anchor questa." La "manus ignota" responsable de ces copies apparaît à plusieurs reprises dans les recueils épigraphiques de Manuzio et notamment dans la transmission des inscriptions de Brescia (MSS Vat. Lat. 5235 I et Vat. Lat. 5244); on la retrouve également dans un des recueils de Johannes

(33) Cité du Vatican, Bibliothèque apostolique vaticane, MS Vat. Lat. 5234, f. 245. Sur Ciceri, on consultera Dirk Sacré, «Een vergeten brief aan Plantin. Fr. Cicereius over P. Manutius, in Ex Officina Plantiniana: studia in memoriam Christophori Plantini (ca. 1520-1589), ed. Marcus de Schepper en Francine de Nave (Anvers: Vereeniging der Antwerpsche Bibliophielen, 1989), 107-20. 
Metellus (Jean Matal, 1517-97) conservé à la Bibliothèque Vaticane (MS Vat. Lat. 6038, ff. 149-203) ${ }^{34}$ et dans un manuscrit conservé à la Bibliothèque Querinienne de Brescia (MS Querin. A.I.4) ${ }^{35}$ et attribué à un érudit local du nom d'Alessandro Totti. ${ }^{36}$ J'avais pensé ainsi pouvoir attribuer à Totti aussi les textes copiés par la même main dans les recueils romains de Manuzio et de Metellus. ${ }^{37}$ Or, dans le MS Vat. Lat. 6038 (f. 154v), l'auteur que je croyais être Totti précise en marge de l'inscription à Fabricia Arethusa qu'il a vu la pierre et que l'on ne peut se fier aux copistes: «cosi l'ho veduto et cavato io. Vedere hora quanto si possa fidar de trascrittori.» Il avait effectivement copié une première fois l'inscription, au f. 155r, avec des erreurs. Quand ce groupe d'inscriptions arrive entre les mains de Metellus, il le collationne avec des copies d'inscriptions de Brescia plus anciennes qu'il conserve dans un autre recueil (MS Vat. Lat. 6039, f. 180v) et au moment de corriger à son tour l'ancienne copie, il traduit en latin la note de l'auteur appelé Totti, mais il désigne cette personne par l'initiale «N.»: "vidit et exscr. (ipsit) $N .{ }^{38}$ L'auteur des recueils de Brescia cités plus haut et de la copie de CIL 6.921 dans le recueil de Manuzio (MS Vat. Lat. 5237, f. 147r) est donc un certain "N.» dont l'écriture se rapproche de celle de Florentius; comme lui, il rédige ses notes épigraphiques tantôt en latin tantôt en italien. "N.» est aussi le seul auteur à nous apprendre la découverte de CIL VI 23592 à la même date que CIL 6.921: "nel sopradetto anno (scil. 1562) fu ritrovata una urna con questa inscrittione." "N.» copie cette inscription au même endroit du recueil de Manuzio que CIL 6.921 (Fig.9). ${ }^{39}$ Le seul autre témoin de l'inscription est Florentius qui précise qu'il s'agit d'une urne: "marmorea urna rotunda alta pedum 1/6,» conservée dans le collection privée de Pantagato ("apud doctiss.(imum) patrem Octavum Pantaghatum»), précisément originaire de Brescia. ${ }^{40}$ Si l'auteur désigné par "N.» devait s'avérer être notre érudit de Haarlem, sa stature prendrait alors plus d'ampleur aux côtés des érudits qui comme Matal, Agustín, Smetius, Pighius, Morillon, Manuzio, Orsini,

(34) La bibliographie sur Matal est rassemblée dans Ginette Vagenheim, "Manus epigraphicae. Pirro Ligorio et d'autres érudits dans les recueils d'inscriptions latines, " (Deuxième Congrès de la Société française d'études néo-latines, Rouen, 10-12 mars 2003), dans L'Italie et la France dans l'Europe latine du XIV'au XVII'siècle. Influence, émulation, traduction, dir. Marc Deramaix et Ginette Vangeheim (Rouen : Presses Universitaires de Rouen et du Havre, 2006): 233-70.

(35) Dans ce recueil ainsi que dans le MS Vat. Lat. 5235 I, seules les indications de lieu sont de la main de main attribuée à Totti.

(36) On consultera la notice relative à ce personnage dans l'index auctorum de CIL 5 et des Inscriptiones Italiae, Brixia.

(37) Ginette Vagenheim, «Appunti sulla tradizione manoscritta delle epigrafi: esempi bresciani di Pirro Ligorio, "Epigraphica 53 (1991): 175-213.

(38) Pour l'exposé de cette question et les planches de ces différents manuscrits, je me permets de renvoyer à Vagenheim, Manus epigraphicae, 238-9. Richard Cooper proposait pour sa part d'identifier cet auteur avec Nicolas Micaut mais sans apporter d'arguments à ce choix: «Epigraphical Research in Rome in the Mid-Sixteenth Century: the papers of Antonio Agustín and Jean Matal, "in Antonio Agustín between Renaissance and CounterReform, ed. Michael H. Crawford (London: University of London. The Warburg Institute, 1993), 95-112.

(39) Manuzio recopiera à deux reprises l'inscription CIL VI 23592 dans le même recueil (f. 174 et f. 181).

(40) Les deux autres copies de CIL 6.23592 conservées dans les manuscrits de Manuzio viennent de Florentius, comme je me propose de le montrer dans une prochaine étude sur la collection de Pantagato, qui paraîtra dans la revue Epigraphica. 


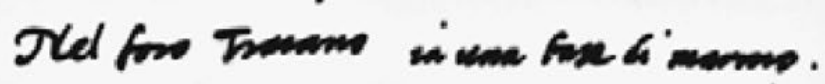

SAT VRNINIO SE CVNDO.V.C PRAESIDI PROVINCLAE AQVTTANILAE MAGISTRO MEMORIAE. COMITI ORQ! $X I S$ PRIMI .PROCONSVLI AFRICAE.TTEM COMITI ORPINIS PRIMI. TNTRA COM SISTORIVM-ET PVAESTORI PRAEF: PRAETGRIO ITERVM OB. E GREGTA EIVS IN REMPVELICAM MERITA RO.NA. VAELENTINIANVS ET VALENS. VICTORESAC TRIVMFA TORES SEMPER AVGVSTI STATVAM SVA.AVR. CONSTI TVI LOCARSCVE IVSSERTNT.

Fig. 9: CIL VI 921 et CIL VI 23592 copiées par «N.». 
Panvinio, Ligorio Pantagato et bien d'autres qui donnèrent leur essor aux études épigraphiques à Rome dans la seconde moitié du seizième siècle. ${ }^{41}$ Mais avant de revenir aux recueils d'inscriptions, il faudra lire les lettres, conservées à la Staats- und Universitätsbibliothek de Hambourg, de Florentius à Pighius et à Torrentius envoyées de Rome entre 1558 et 1567, qui contribueront sans aucun doute à donner plus de vie à un personnage connu aujourd'hui uniquement comme un homo epigraphicus.

(41) Un bon panorama de ces recherches est présenté par William Stenhouse, Reading Inscriptions and Writing Ancient History: Historical Scholarship in the Late Renaissance (London: Institute of Classical Studies, 2005). 